\title{
EXTENDED BASELINE PHOTOMETRY OF RAPIDLY CHANGING WEATHER PATTERNS ON THE BROWN DWARF BINARY LUHMAN-16
}

\author{
R. A. Street ${ }^{1}$, B. J. Fulton ${ }^{1,2}$, A. Scholz ${ }^{3}$, Keith Horne ${ }^{3}$, C. Helling ${ }^{3}$, D. Juncher ${ }^{3}$, G. LeE ${ }^{3}$, and S. Valenti ${ }^{1}$ \\ ${ }^{1}$ LCOGT, 6740 Cortona Drive, Suite 102, Goleta, CA 93117, USA; rstreet@lcogt.net \\ ${ }^{2}$ Institute for Astronomy, University of Hawaii, 2680 Woodlawn Drive, Honolulu, HI 96822-1839, USA \\ ${ }^{3}$ School of Physics and Astronomy, University of St Andrews, St Andrews, Fife, KY16 9SS, UK \\ Received 2014 April 15; accepted 2015 September 10; published 2015 October 20
}

\begin{abstract}
Luhman-16 (WISE J1049-5319) was recently discovered to be a nearby ( 2 pc) brown dwarf binary that exhibits a high degree of photometric variability $(\Delta m \sim 0.1 \mathrm{mag}$ ). This is thought to be due to the evolution of "cloud" features on the photosphere, but Luhman-16 has been found to show unusually rapid changes, possibly resulting from fast-evolving "weather." This target is of particular interest because it consists of a co-evolutionary pair of brown dwarfs spanning the transition between $\mathrm{L}$ and $\mathrm{T}$ spectral types (L7.5 and T0.5), which are expected to be associated with changes in cloud surface coverage. Being comparatively bright $(I \sim 15.5 \mathrm{mag})$, the target is well suited for observation with the new Las Cumbres Observatory Global Telescope Network (LCOGT) of $1 \mathrm{~m}$ telescopes. We present long-time baseline photometric observations from two of LCOGT's southern hemisphere sites, which were used in tandem to monitor Luhman-16 for up to $13.25 \mathrm{hr}$ at a time (more than twice the rotation period), for a total of 41.2 days in the SDSS-i' and Pan-STARRS-Z filters. We use this data set to characterize the changing rotational modulation, which may be explained by the evolution of cloud features at varying latitudes on the surfaces of the two brown dwarfs.
\end{abstract}

Key words: brown dwarfs - stars: general - stars: individual (Luhman-16, WISE-J1049-0053) - stars: low-mass

\section{INTRODUCTION}

Luhman (2013) recently discovered the brown dwarf binary WISE J104915.57-531906.1 at a distance of $2.0 \mathrm{pc}$, making it the third closest system to the Sun. The object was found through multi-epoch photometry in the WISE database (Wright et al. 2010) and was also detected in the catalogs from 2MASS (Skrutskie et al. 2006), DENIS (Epchtein et al. 1999), DSS (Luhman 2013), IRAS, AKARI (Ishihara et al. 2010), and GSC (Mamajek 2013). The two components of the binary have a projected separation of 3.1 AU and spectral types of L7.5 and T0.5 (Burgasser et al. 2013; Kniazev et al. 2013), with $T_{\text {eff }}=1310 \pm 30$ and $1280 \pm 75 \mathrm{~K}$, respectively (Faherty et al. 2014). This pair, with the short-hand name "Luhman-16," is one of a series of discoveries of very nearby brown dwarfs, following the publication of objects between 3 and $6 \mathrm{pc}$ (McCaughrean et al. 2004; Artigau et al. 2006; Lucas et al. 2010; Scholz et al. 2011).

This very short distance, combined with the fact that both components are close to the $\mathrm{L} / \mathrm{T}$ transition regime in terms of their spectral type, makes Luhman-16 an intriguing test case for models of brown dwarf atmospheres. The cool atmospheres of brown dwarfs allow the condensation of metal-rich dust grains, which form clouds and provide an additional source of opacity (Tsuji et al. 1996). Below effective temperatures of $2000 \mathrm{~K}$, dust clouds significantly alter the temperature and pressure structure in the atmosphere and as a result, the colors and spectra of the objects (Witte et al. 2009).

While clouds in brown dwarfs may seem like a nuisance for those who aim to determine the fundamental parameters of these objects with great accuracy, for others they are an interesting environment for investigating physical mechanisms that may also affect the atmospheres of giant extrasolar planets (Helling et al. 2008b). There is already evidence that the spectra of planetary-mass companions around nearby stars and brown dwarfs can only be explained with the help of sophisticated cloud models (Barman et al. 2011a, 2011b; Skemer et al. 2011; Oppenheimer et al. 2013).

Over the past decade several independent groups have developed models of dust formation in brown dwarf atmospheres with varying simplifications, parameterizations, and assumptions (Ackerman \& Marley 2001; Allard et al. 2003; Woitke \& Helling 2003; Burrows et al. 2006, e.g.,). For a more detailed discussion of these cloud models, we refer to Helling et al. (2008a). Compared with the observations, several existing models agree reasonably well with the colors and spectra of L dwarfs, down to temperatures around $1600 \mathrm{~K}$ (Witte et al. 2011, e.g.,). The next challenge is to interpret the phenomena observed at even cooler temperatures, in particular at the $\mathrm{L} / \mathrm{T}$ transition.

At this boundary, the observations indicate that the cloud properties undergo a fundamental change. This is evident from two empirically established findings.

(1) The $J-K$ near-infrared colors of brown dwarfs show a clear discontinuity around the $\mathrm{L} / \mathrm{T}$ transition and turn sharply toward the blue. This is related to the so-called "J-band bump" (Tinney et al. 2003), a brightening in the J-band toward cooler temperatures. Luhman-16 shows this behavior exactly, with the cooler component being brighter in the $J$-band by $0.3 \mathrm{mag}$ and bluer in $J-K$ by $0.6 \mathrm{mag}$ (Burgasser et al. 2013). While this behavior can partly be attributed to a global change of the cloud layer (for example, clouds sinking below the photosphere or a more efficient rain-out of the clouds), Burgasser et al. (2002) and others suggested that the sharpness of the transition requires a break-up of the cloud coverage.

(2) Several objects around the $\mathrm{L} / \mathrm{T}$ transition show pronounced quasi-periodic variability (Artigau et al. 2009; Radigan et al. 2012, 2013; Girardin et al. 2013; Heinze et al. 2013, 2015; Buenzli et al. 2014), whereas most L dwarfs are somewhat less variable (Bailer-Jones \& Mundt 2001; Gelino et al. 2002; Koen 2013, e.g.,). Observations to date have 
supported model where patchy or inhomogeneous cloud, carried in and out of view by the object's rotation, is the cause of this variability (Burgasser et al. 2002; Marley et al. 2010).

Radigan et al. (2012) used linear combinations of onedimensional (1D) atmospheric models matched to the NIR spectrum and time series photometry of 2MASS J21392676 +0220226 (2M2139) and concluded that the atmosphere contained either a thick, cooler cloud overlaying a thinner cloud deck or regions of high condensate opacity interspersed with warmer ( $\Delta T_{\text {eff }} \sim 175-425 \mathrm{~K}$ ) regions of lower opacity. This was supported by a study of times series spectra of 2M2139 and SIMP0136 (2MASS J0136565+093347) by Apai et al. (2013), who found a model with two spectral components with different cloud properties and effective temperatures were required to fit the observations. Both authors ruled out a model where holes in the cloud were cleared entirely, favoring instead a thin/thick cloud scenario (see Apai et al. 2013, Figure 6). These variations are often interpreted as "weather" on brown dwarfs, although we note that this is an observational bias in the following sense: L dwarfs have so much dust that we might not see any variability, whereas in T dwarfs the dust may form so deep inside the atmosphere that the gas alone is optically thick enough to prevent observation of intrinsic cloud variability.

In summary, the $\mathrm{L} / \mathrm{T}$ transition is expected to be associated with cloud patchiness, which can be probed by studying the photometric variability, and the proximity of the Luhman-16 binary, with co-evolutionary components spanning this transition make it an excellent target for observation.

Earlier photometric monitoring campaigns for Luhman-16 have indeed shown that the object is strongly variable. Gillon et al. (2013) and Biller et al. (2013) demonstrated that while both components are variable to some degree, the T0.5 dwarf displays the greatest amplitude. Buenzli et al. (2014) obtained a time series of spatially resolved NIR spectra on both binary components with the Hubble Space Telescope (HST)/WFC3 which supported a two-layer cloud model of warm $\left(T_{\text {eff }}=1300 \mathrm{~K}\right)$, thinner cloud and a cooler $\left(T_{\text {eff }}=\right.$ $1000-1100 \mathrm{~K})$, thicker cloud, with out-of-equilibrium atmospheric chemistry. They were also able to compare models for Luhman-16A (which was consistent with a single cloud layer model) with Luhman-16B, finding different effective temperatures and sedimentation efficiencies across the $\mathrm{L} / \mathrm{T}$ transition. Crossfield et al. (2014) graphically reinforced this model by mapping the surface of Luhman-16B by means of Doppler Imaging analysis. They obtaining a time series of Very Large Telescope $(\mathrm{VLT}) /$ CRIRES spectra over the course of $\sim 1$ rotation cycle and observed bright and dark regions rotating in and out of view, which they interpreted as a snapshot of the clouds during that rotation cycle. Osten et al. (2015) looked for radio and X-ray emission from Luhman-16 without success from the Australia Telescope Compact Array and Chandra facilities, respectively. They inferred upper limits on the maximum size of any coherent radio emitting region to $<0.2 \%$ of the brown dwarf's radius or $<20 \%$ of the radius for incoherent radio emission.

While the light curves presented by Gillon et al. (2013) and Buenzli et al. (2014) clearly show a periodicity of close to $5 \mathrm{hr}$, presumably corresponding to the rotation period, they also show rapid evolution on timescales of days or less, possibly indicating fast-evolving weather patterns. To characterize the atmosphere of this benchmark object and its evolution, continuous monitoring over many consecutive rotational cycles is needed. In this paper, we present monitoring of Luhman-16 using the Las Cumbres Observatory Global Telescope Network (hereafter LCOGT). Our focus is to characterize the evolving light curve of this extraordinary object in two optical bands. These observations were coordinated with Burgasser et al. (2014), and overlap their intensive, multi-instrument monitoring campaign. They obtained contemporaneous measurements on 2013 April 26 UTC consisting of a 45 minute time series of NIR spectra from IRTF/SpeX and photometry (spanning $7.5 \mathrm{hr}$ ) from ESO/TRAPPIST using a broadband $\mathrm{I}+\mathrm{z}$ filter. Both data sets exhibit clear variability from the B-component of the binary. That study measured the colder spots of the atmosphere to cover $\sim 30 \%-50 \%$ of the surface, varying by $15 \%-30 \%$ over the rotation period.

Burgasser et al. (2014) related the variation in spot coverage to the maximum fractional feature size predicted by the Rhines scaling relation (Rhines 1970), which was derived from jet features in solar system giant planets. Assuming the same relation holds for the atmospheres of brown dwarfs, they inferred wind speeds on Luhman-16B between 1.6 and $3.4 \mathrm{Km}^{-1}$ for "hot regions" of $1700 \mathrm{~K}<T_{\text {hot }}<1900 \mathrm{~K}$. This implies advection timescales of $\tau_{\mathrm{adv}} \sim(2-5) \times 10^{4} \mathrm{~s} \sim$ 1-3 rotation cycles, consistent with the timescales of the variation seen in the light curve.

This work explores the evolution of the variability of this fascinating object over timescales much longer than the rotation period. We describe our observations and data reduction in Sections 2 and 3 and present an analysis of the variability over different timescales in Section 4. In Section 5 we discuss our findings in context of the causes of the variability.

\section{OBSERVATIONS}

LCOGT operates a network of 1 and $2 \mathrm{~m}$ telescopes distributed across the globe in both hemispheres (for a complete description, see Brown et al. 2013). The $1 \mathrm{~m}$ telescopes in the southern hemisphere are organized in clusters, and this program made use of the three telescopes at the CerroTololo Interamerican Observatory (CTIO, Chile), and the three at the South African Astronomical Observatory (SAAO, South Africa). Two telescopes have also been installed alongside LCOGT's 2 m telescope (Faulkes Telescope South, FTS) at the Siding Spring Observatory in Australia, but were not available at the time of these observations, while FTS was offline for an extended period due to mirror realuminizing. All of the telescopes are robotically operated, and at the time of the observations (during the network's 2013 commissioning period), all of the $1 \mathrm{~m}$ telescopes hosted SBIG STX-16803 cameras with Kodak KAF-16803 front-illuminated $4096 \times$ 4096 pix CCDs, used in bin $2 \times 2$ mode. The $1 \mathrm{~m}$ telescopes are designed to be as identical as possible to facilitate networked observations, and all feature the same complement of filters. These observations were made in both SDSS- $\mathrm{i}^{\prime}$ and Pan-STARRS-Z due to the very red nature of the target.

The availability of telescopes at multiple sites, and multiple identical telescopes at each site make the LCOGT network an extremely powerful tool for time domain astronomy. For the purposes of this project we exploited two distinct observing modes.

To capture the short-term (hours-days) evolution of the target's light curve, we scheduled continuous, simultaneous 

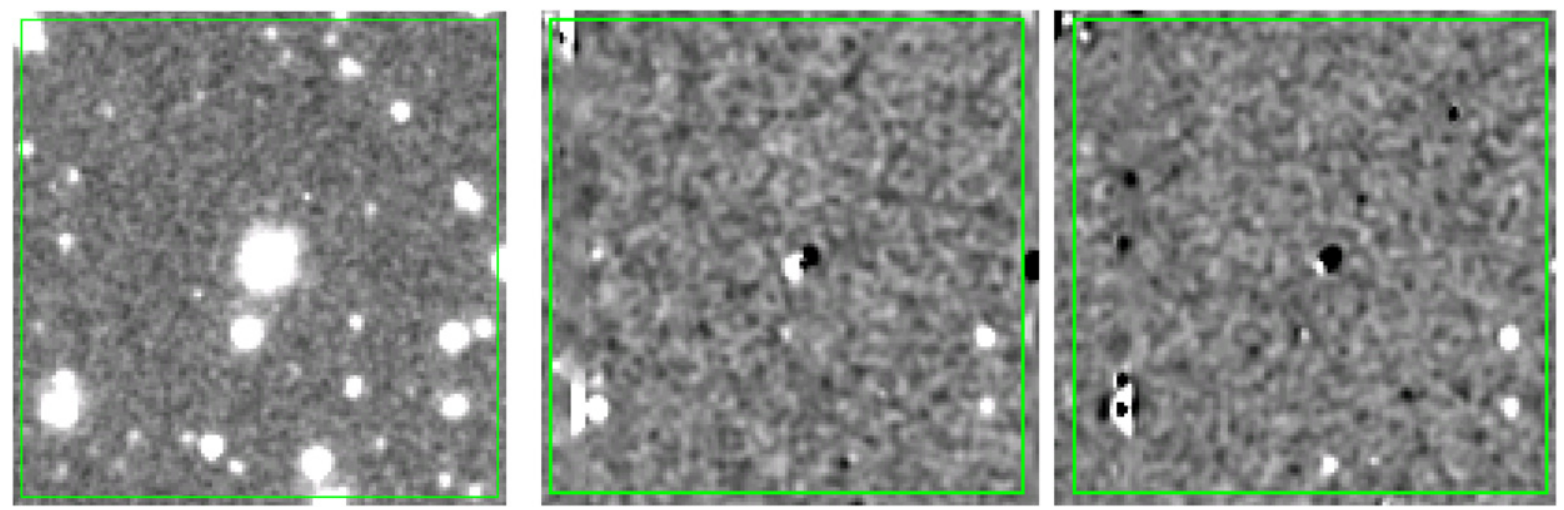

Figure 1. (Left): Reference image in the Pan-STARRS-Z band, centered on Luhman-16 with a 1 arcmin square field of view. (Right): Difference images, also in the Pan-STARRS-Z band and centered on Luhman-16, from the LCOGT-Chile, Dome C data set. The green box indicates a 1 arcmin square field and all images are oriented with north up and east to the left. These images show the subtracted frames for some of the best seeing images obtained: 1.17 arcsec on 2013 April 09 (left) and 1.27 arcsec on 2013 April 16 (right). The changing PSF of Luhman-16 is due to the varying light contributed by both components.

imaging on two parallel telescopes at each site, one in each filter, for as long as the object was visible from that site. At SAAO we observed between 17:00-00:30 UTC and at CTIO between 23:00-06:15 UTC each night for multiple nights backto-back (2013 April 18-21, April 23-26 UTC), resulting in a light curve with continuous (imaging every $\sim 2$ minute) segments of up to $13.25 \mathrm{hr}$.

In order to explore periodicities and variability on timescales of days-weeks, we also performed "monitoring" observations where a single telescope would take sets of exposures in alternating filters. These observation groups were then slotted into gaps in the telescope schedule between other programs wherever possible. Though at lower and irregular cadence, these observations have a much lower impact on other programs and can therefore continue for much longer. We gathered data in this mode between 2013 April 11-May 20 UTC.

\section{DATA REDUCTION}

Preprocessing (bias, dark, and flat-field correction) was performed by LCOGT's Standard Pipeline, which is based around ORAC-DR recipes (Cavanagh et al. 2008).

The 1.5 arcsec angular separation of the Luhman-16 component stars is close to the median full width at half maximum (FWHM) of the point-spread function (PSF) in most of these data sets, making it difficult to separate the independent variability of the stars. Since the seeing at LCOGT's Chilean site is often better than at other sites ( $\sim 1.1-1.2$ arcsec), we attempted to derive photometry for two of the best data sets using the DanDIA (Bramich 2008) package to apply Difference Image Analysis techniques. The reference image selected is shown in Figure 1, and difference images from some of the best seeing frames are also shown. Upon extracting the light curves of both component stars in PanSTARRS-Z and SDSS-i', it became clear that the variability observed in both was highly correlated.

This happens in DIA analysis when the PSFs of two variables overlap and the PSF fitting performed on the difference images only fits one star at a time, which is the case with DanDIA (see V52 and V53 in Arellano Ferro et al. 2012). In the case of Luhman-16, there is the added complication that the binary has a high proper motion and parallax (0.496 \pm 0.037 arcsec, Luhman 2013), and will have moved $\sim 0.2$ arcsec over the course of our observations. Hence, the PSF model to fit to the difference images requires a simultaneous PSF fit at two positions that also change over the course of the observations. For our data sets with poorer seeing, these problems are further exacerbated. We therefore chose to pursue aperture photometry to reduce these data, effectively taking the Luhman-16 binary as a single object. However, from visual inspection of the difference frames, we note that the highest degree of variability appears to come from the southeastern star during this period.

The data were then reduced with two independent aperture photometric pipelines to provide a consistency check, both employing implementations of DAOphot to derive aperture photometry (Stetson 1987). Although LCOGT's cameras are designed to be as homogeneous as possible, the images from each telescope/camera combination were reduced separately to ensure the correct gain and read noise properties were applied for each camera. We selected an initial set of reference stars by manual inspection and derived differential light curves of Luhman-16 for each of the six cameras.

Differential photometry did a reasonable job of removing the signature of changing atmospheric extinction over the course of an individual data set. However, when the light curves in each filter were plotted together, magnitude offsets of the order of $\Delta m \sim 0.1$ mag were evident between them.

A number of authors have discussed techniques for obtaining high-precision differential photometry, notably transiting planet search teams. The most common case is light curves from a single observatory (e.g., WASP, Kepler, TRAPPIST) that can span days to many months. Data from LCOGT's network differs from these cases due to two factors: (1) the field of view is small when compared with a survey such as WASP, and it cannot be safely assumed that a significant number of nonvariable stars with cataloged brightness and color data will be present in every field and (2) LCOGT can have multiple cameras at longitudinally distributed sites observing the same target through different airmasses. These data sets may or may not include data taken simultaneously.

We explored a number of avenues for combining data from different telescopes, including the sYsREm (Tamuz et al. 2005) and TFA (Kovács et al. 2005) algorithms, and the PDC-MAP technique used by the Kepler team (Smith et al. 2012). The essential approach is to recognize that the raw (non-differential) light curves of all stars within the field of view are dominated 

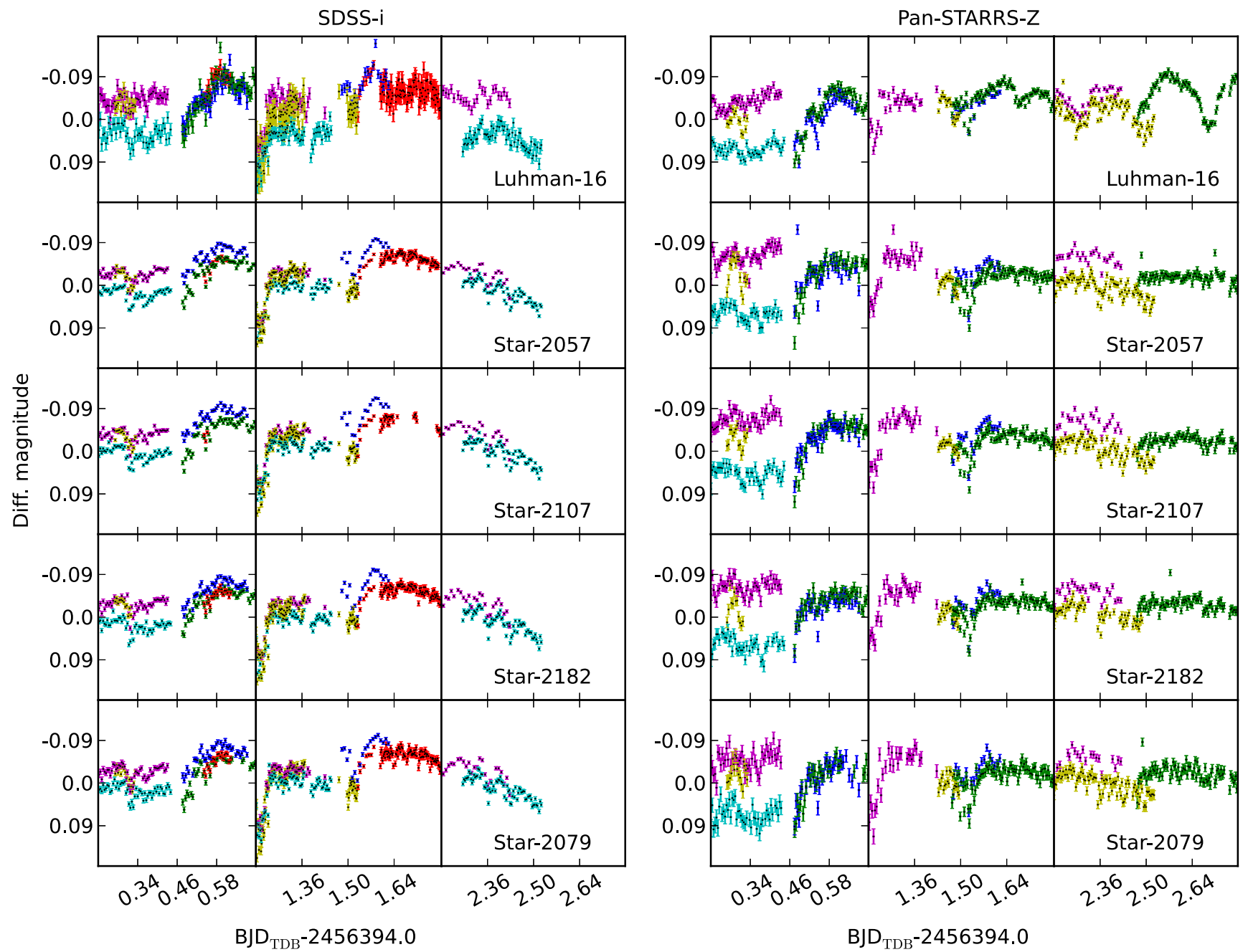

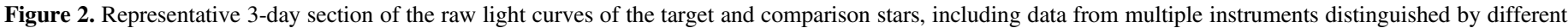
colors.

by trends due to a number of atmospheric and instrumental effects, giving even constant stars the same apparent variability. Each algorithm aims to select a set of stars that are not intrinsically variable to the precision of the data and use these stars to empirically determine the time-dependent trends in the data. Once identified, these trends can then be removed from all light curves in the data set, but care must be taken to avoid mistaking genuine variability for an instrumental artifact.

Our approach was not to distinguish the instrumental effects due to different nights on the same camera from those due to observations taken with different cameras. Instead we simply attempt to identify common systematic trends. Therefore, for each star, we concatenated all data sets in a given filter to produce a set of combined raw light curves.

The mean magnitude and rms scatter was first computed for all combined raw light curves, weighted by the inverse variance of each data point's measurement error. Of the 2466 stars measured, we restricted our choice of comparison stars to those that had at least the same number of valid measurements as the target, and had instrumental mean magnitudes within $\sim 4$ mag of that of the target. At this stage, all light curves were dominated by instrumental trends and the rms of the stars was fairly constant with magnitude, so we used a $3 \sigma$ cut on rms to reject any stars showing obvious variability over and above that. This produced a preliminary list of 13 comparison stars.

After subtracting the mean flux from each comparison star light curve, we calculated the mean of the comparison star residual fluxes for each image. The resulting time series represents the combined trends common to all light curves. The light curves of both target and comparison stars were divided by this supercomparison, and normalized by their mean flux, computed by weighting each data point by its photometric errors. The differential light curves of the comparison stars were then inspected to weed out any showing signs of variability. After each rejection, the differential photometry was re-computed and the process was iterated until a satisfactory set of four stars was reached. This procedure was carried out using the Pan-STARRS-Z data and then the same set of stars was then used to derive differential photometry from the SDSS- $i^{\prime}$ light curves. Figures 2 and 3 compare a representative section of the raw light curves for our target and comparison stars with the same data section, post-differential photometry. Table 1 provides the details of all comparison stars, and their distribution within the reference image is shown in Figure 4.

To test how well data from different telescopes were combined, we plotted the resulting light curves for the target 

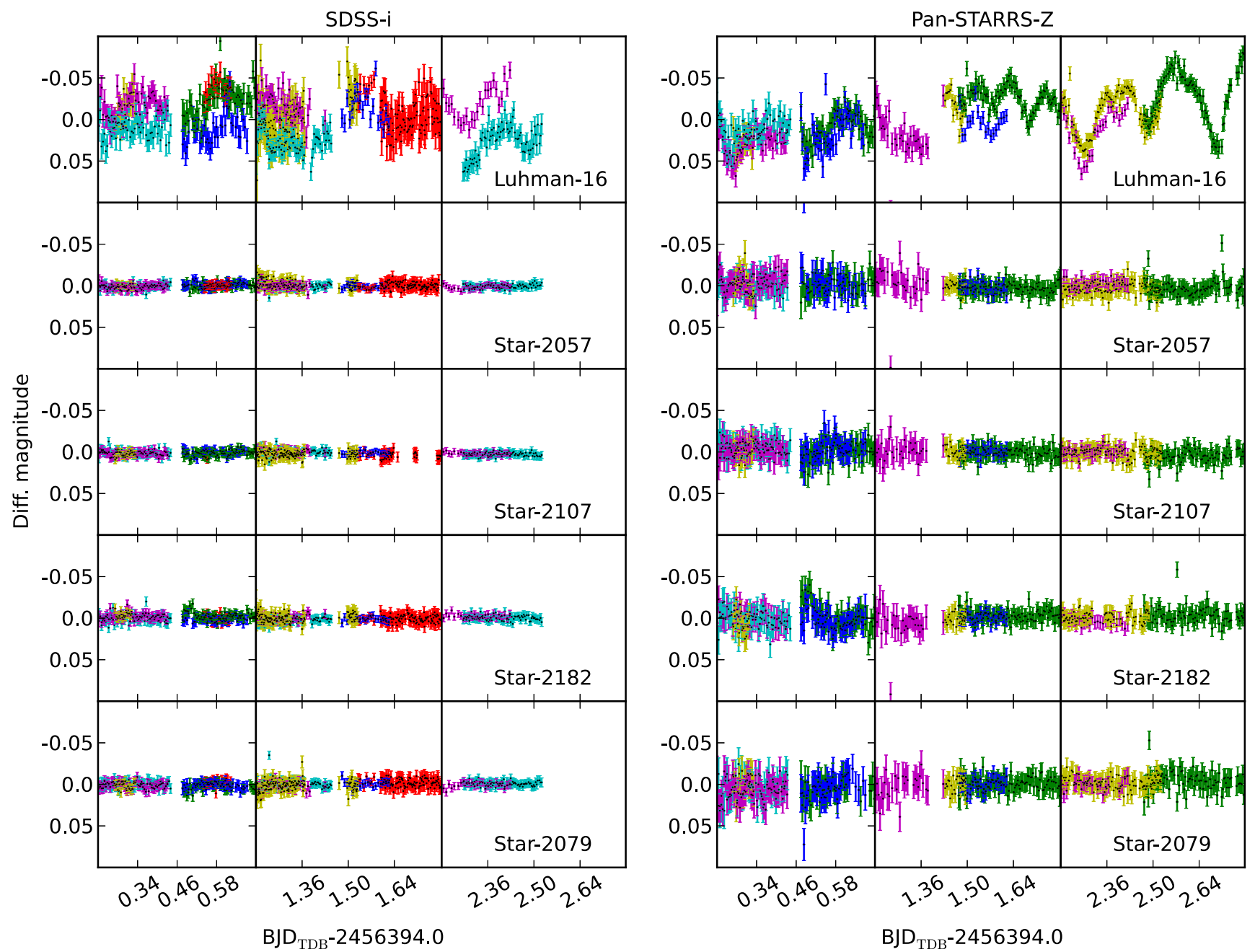

Figure 3. Same 3-day section of data as in Figure 2 of the differential photometry for the target and comparison stars, including data from multiple instruments distinguished by different colors. The residual bulk offsets described in the text can be seen in the light curves.

Table 1

Details of the Target and Comparison Stars

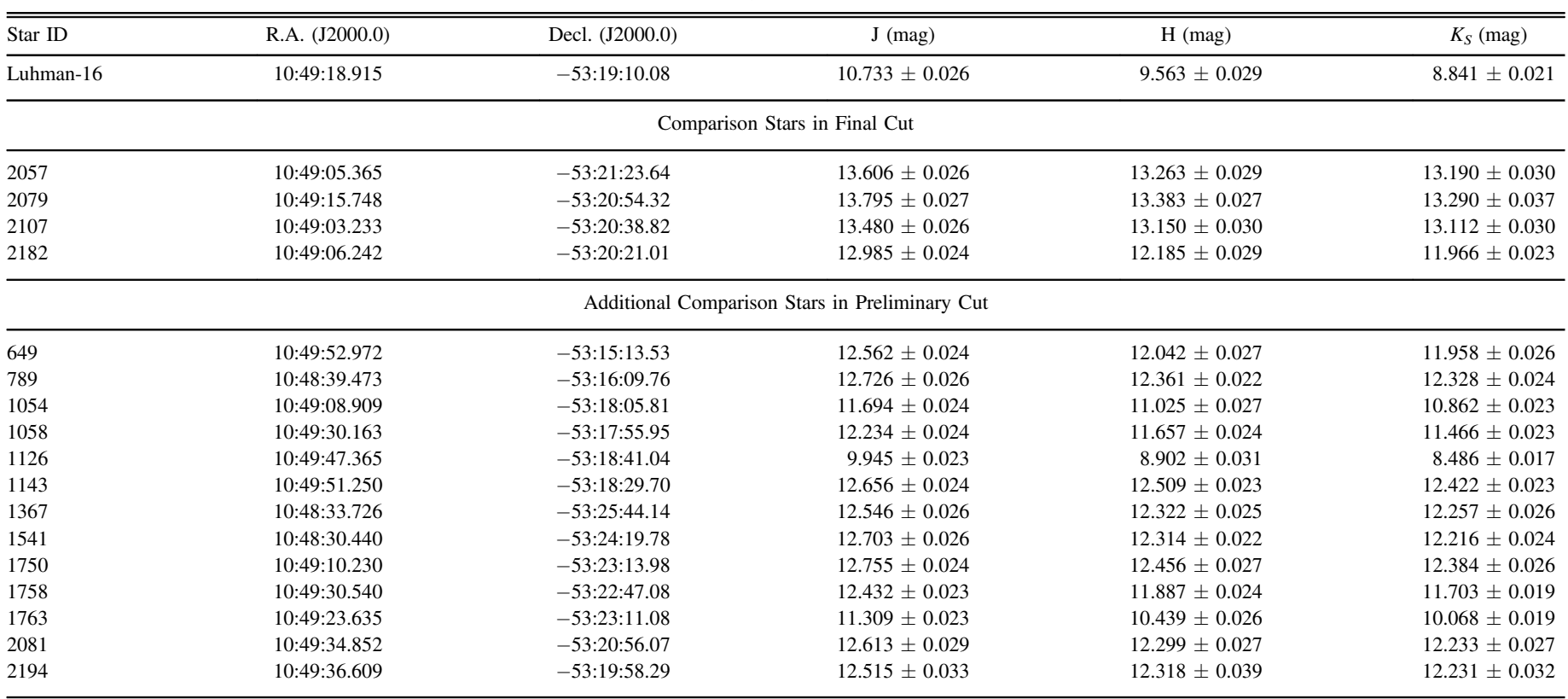

Note. The coordinates and magnitudes are derived from the 2MASS point source catalog (Skrutskie et al. 2006). 

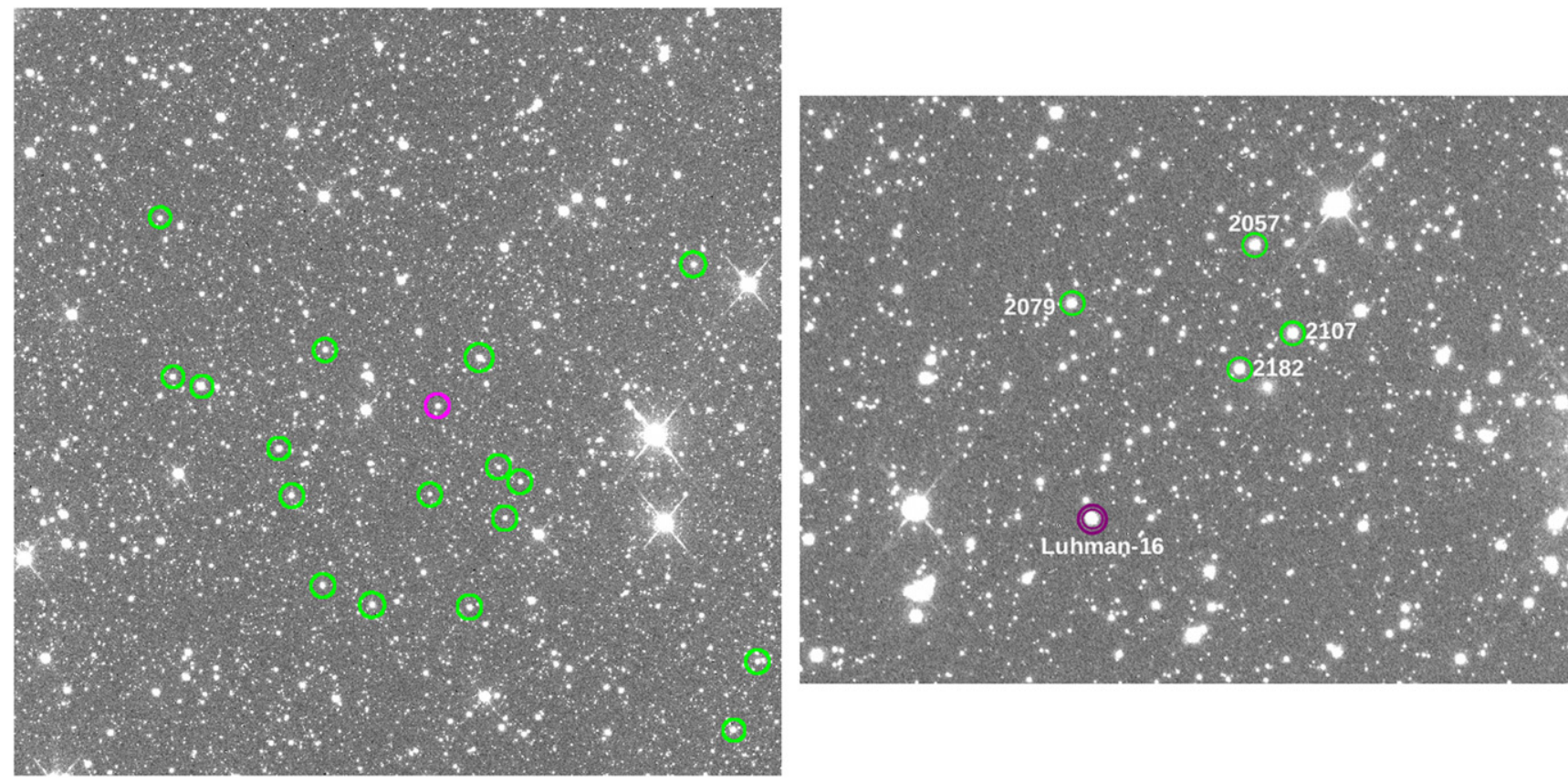

Figure 4. Distribution of the comparison stars in the Pan-STARRS-Z reference image. (Left): The preliminary selection of stars. (Right): The final selection. Luhman16 is in the center in the left image and is indicated with a different color ring in both images.
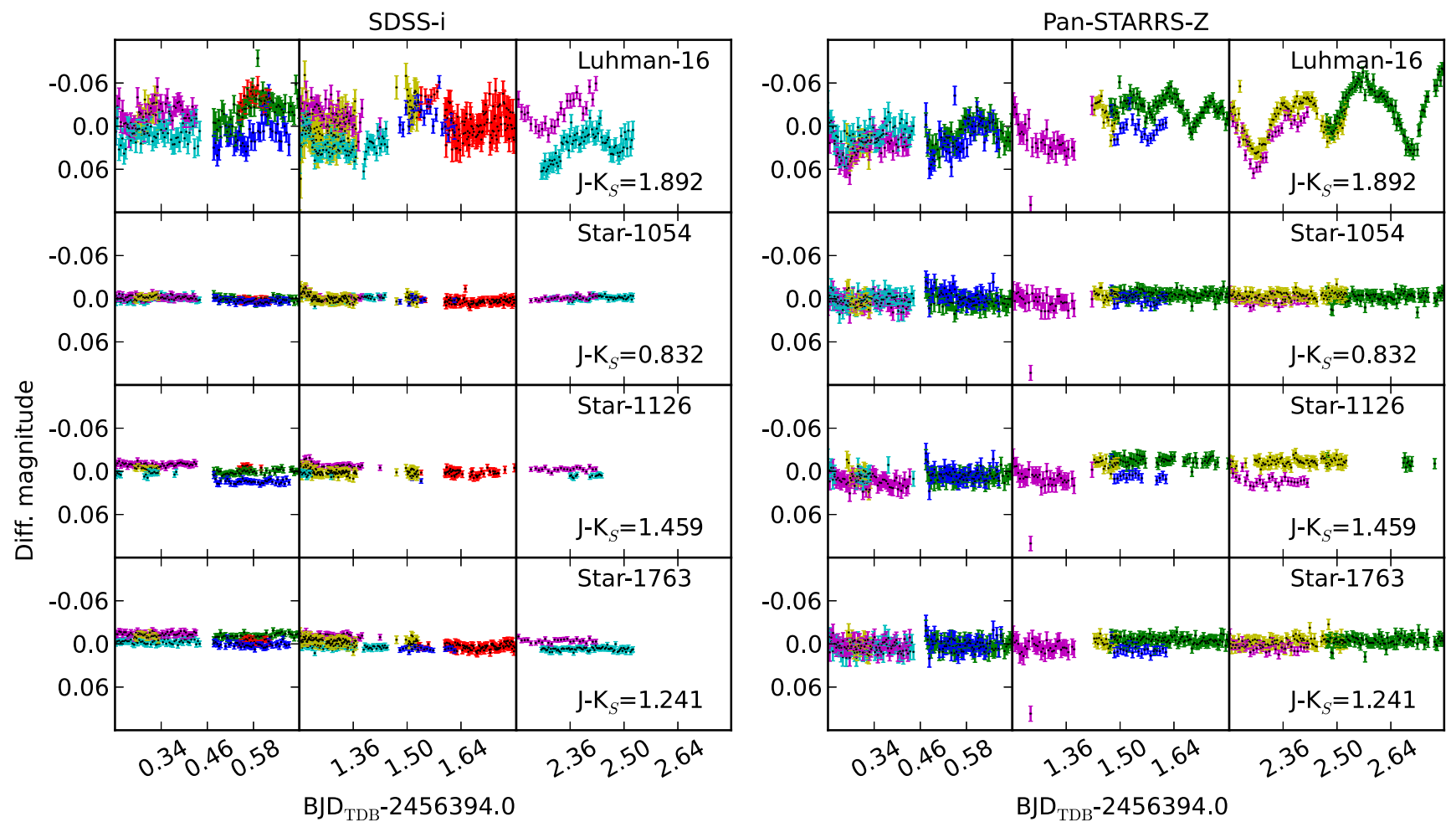

Figure 5. Same 3-day section of data as in Figure 2 of the differential photometry for the target and a selection of field stars of different $J-K_{S}$, showing the residual offsets between data from multiple instruments (distinguished by different colors).

and a selection of field stars of different $J-K_{S}$, distinguishing the data from different instruments, in Figure 5. This revealed small $(<0.02 \mathrm{mag})$ residual offsets between simultaneous data in the same filter from different telescopes, including data from telescopes at the same site.

Differential photometry generally corrects for photometric trends due to atmospheric absorption to first-order, accounting for the decrease in apparent magnitude with increasing airmass, but since the absorption is a function of wavelength, there is a second-order term dependent on star color. For a star as red as Luhman-16 we would expect this term to be significant. However, simultaneous data sets in the same filter from telescopes at the same site are taken with effectively the same airmass, so this alone does not explain the offsets. Other possible causes of the residual offsets include the different instrumental (flat-field) signatures of the different 

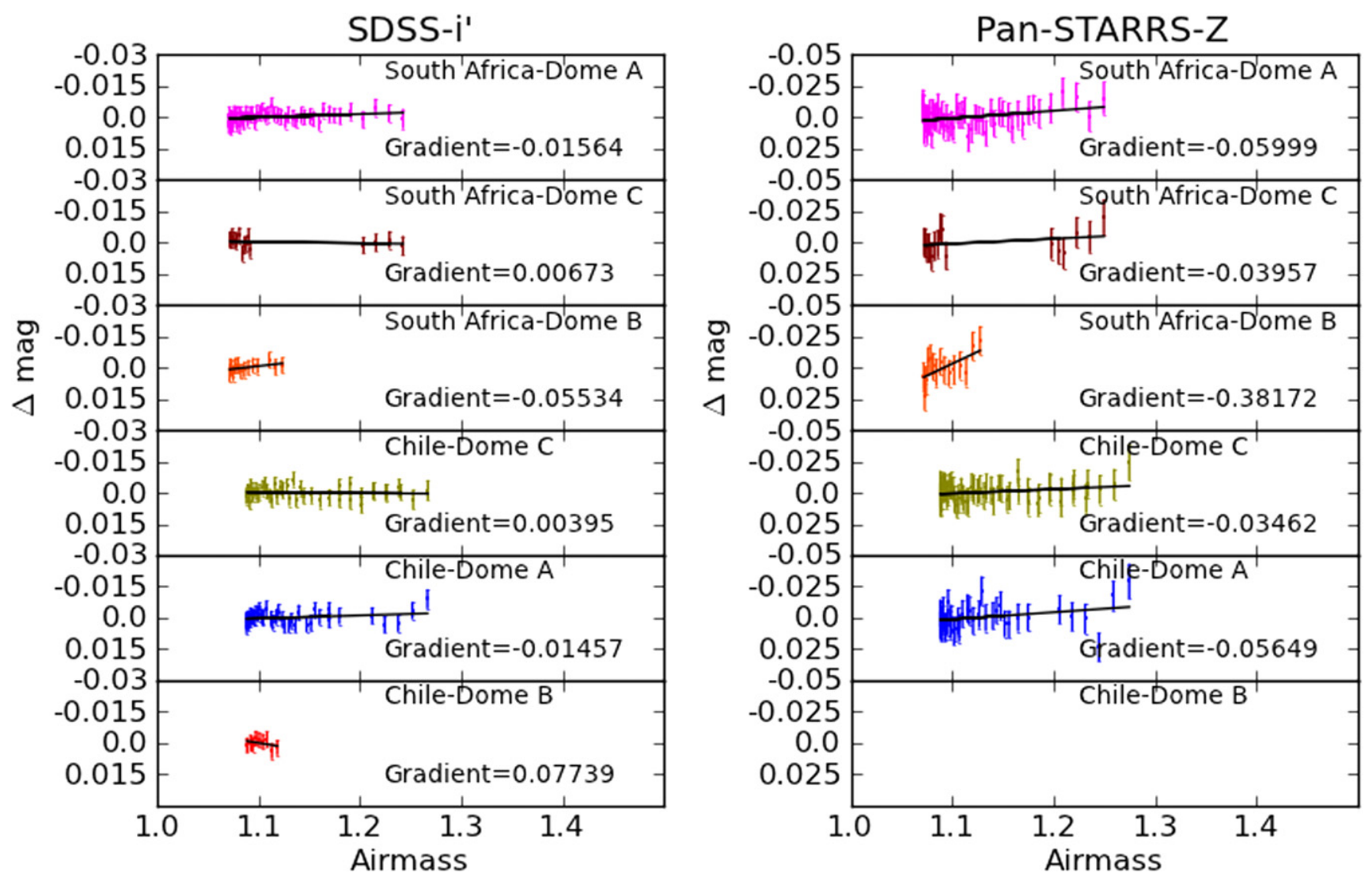

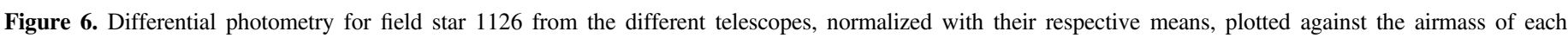
measurement.

cameras, and the variation in pixel position of each star in each frame.

Looking for the cause of these offsets, we searched for correlations between these factors and the residuals of differential light curves (the light curves normalized by their own means) for a set of field stars distributed across the CCD frame and in $J-K_{S}$ color. We used the "preliminary comparison star" set (excluding the final comparison stars) given in Table 1 for this analysis, concentrating on the 1-3 day sections of high cadence data taken in consistently good conditions with multiple telescopes. This provides data spread over a range of airmass and telescope pointing while excluding periods of poor weather.

Figure 6 shows the differential residuals as a function of airmass for the different telescopes for the field star closest to Luhman-16 in color. We fitted a linear function of airmass to the data to each data section. In general we find very small overall gradients in SDSS-i' but consistently higher gradients in Pan-STARRS-Z, a symptom of second-order atmospheric extinction. Similar plots were produced for bluer field stars, which showed lower gradients. This is reinforced by Figure 7, which shows an increase in the standard deviation of the differential residuals for redder stars. This is not unexpected, since the comparison stars are necessarily bluer than the target. We note that the amplitude of this residual is still lower than the amplitude of the variability of the target by a factor $\sim 2.5$, but since no non-variable star of similar color to Luhman-16 was available for direct comparison, we performed additional tests to look for signs of correlations between the photometric residuals and observational and instrumental parameters.
We searched for correlations between the photometric residuals, airmass, and CCD pixel position. We found strong correlations between the pixel positions with airmass, implying that the movement of the telescopes toward higher airmass causes the pointing to change somewhat. This may be the result of slight imbalances in the weight distribution of the telescopes. The stars typically move up to $\sim 40$ pix over the CCD during the nights but additionally shift \pm 25 pix between one night's data and the next. Since different regions of the CCD have different sensitivities at different wavelengths, this introduces a source of color-dependent photometric variation. More sophisticated pointing and guiding algorithms are under development by LCOGT's software team to help eliminate this source of red noise.

LCOGT's normal procedure is to flat-field each frame with the master flat-field closest to it in date. Frequently this means that the image data for a night are flat-fielded with the same master flat-field, but of course different master flats for each camera. This could also contribute to night-to-night offsets between data taken with different cameras.

Nevertheless, a star may be assumed to show the same behavior at any given time in data taken with the same filter, meaning that the simultaneous single-color light curve segments should overlap in a differential light curve whose mean is normalized to zero.

We therefore developed a second-stage algorithm that took each single-filter differential light curve from the first stage and separated the data from different cameras. As simultaneous light curve segments do not consist of frames taken at precisely the same instant, the per-camera light curves were binned such that they were sampled at the same time intervals with a 

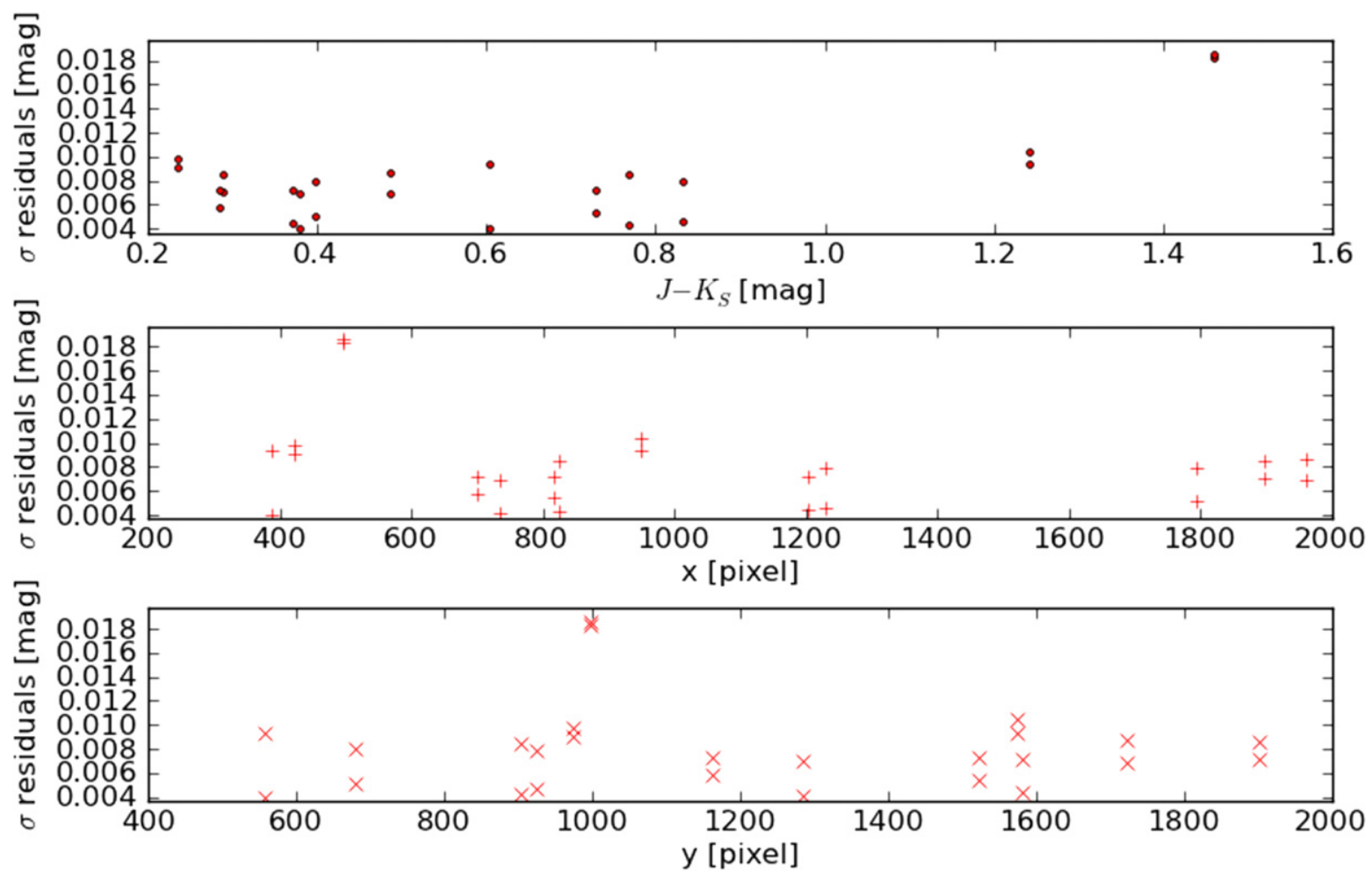

Figure 7. Standard deviation, $\sigma$, of differential light curves for a set of field stars distributed across the image and in $J-K_{S}$ color.

30 minute cadence. The code then calculates the mean bulk offset per segment between all data points in contiguous, overlapping sections of data (typically several hours long), applying the correction to the light curve segment furthest from the overall differential mean of zero.

Following this step, it is advisable to verify the data reduction strategy and to quantify the residual noise in the light curves. This is usually described as a combination of "white" (uncorrelated) and "red" (correlated) noise. The latter term is particularly important as it results from noise sources that cause apparent trends in the light curves that could potentially mimic a true signal or produce misleading parameters when superimposed on intrinsic variability.

In Figures 8-10, we plot the target's final differential light curve as functions of airmass, pixel position, and the average FWHM of stars in each frame as a proxy for the seeing. That the target's light curve variation is intrinsic and not a symptom of systematic trends is supported by the lack of correlation between these parameters and the photometric residuals.

Our aim is to measure the intrinsic periodicity(ies) of Luhman-16, if any. The most natural approach is to produce a power spectrum (squared variability amplitude) from the light curve, provided the intrinsic signal can be distinguished from the noise. Vaughan (2012) presents a good review of a range of techniques for time series analysis as applied in many different fields of astronomy, and discusses the recovery of reliable noise power spectra from sparsely sampled time series.

The analysis of Luhman-16 is particularly challenging because the variability is both quasi-periodic and changes on timescales that are short relative to the periodicity itself. This rules out the application of many commonly used techniques such as, for example, the Bartlett method Bartlett (1948), which relies on computing a series of periodograms at intervals along the light curve and taking the average. As we show in
Section 4, Luhman-16 is non-stationary (the periodicities vary) on the shortest timescales for which we are able to estimate power spectra.

A great deal of work in the analysis of red noise in light curves has been derived from exoplanet transit work, following from Pont et al. (2006). Their technique depends on excluding data taken during transits from the target light curve, then analyzing the noise in the remaining photometry, under the assumption that the rest of the light curve should maintain constant brightness out of transit. This is problematic to apply directly to Luhman-16 because the photometric variations are continuous and unpredictable, making it difficult to distinguish systematic noise sources from intrinsic variability. However, our differential photometry depends on the assumption that our reference stars are constant throughout the observations. The data on these stars, which span a range of colors, were subjected to the same observation conditions, instrumentation, and data reduction process, and therefore should reflect similar systematics.

To quantify the red noise in our data, we followed a similar procedure to Pont et al. (2006) for the light curves of each of our reference stars. They relate the uncertainty on the signal amplitude, $\sigma_{d}$, as $\sigma_{d}=\nu^{1 / 2}(n)$, where

$$
\nu(n) \equiv \frac{1}{n^{2}} \sum C_{i j}
$$

and $C_{i j}$ are the covariance coefficients between the $i$ th and $j$ th measurements. $\nu(n)$ is estimated from the variance of the average of $n$ data points within time interval $l$, which is free from any known signal.

1. We calculated the mean flux $\left(F_{j}\right)$ within sliding intervals $\left(j=1 \ldots N_{j}\right)$ of duration $l$, which progress through each light curve in steps smaller than the time-sampling 

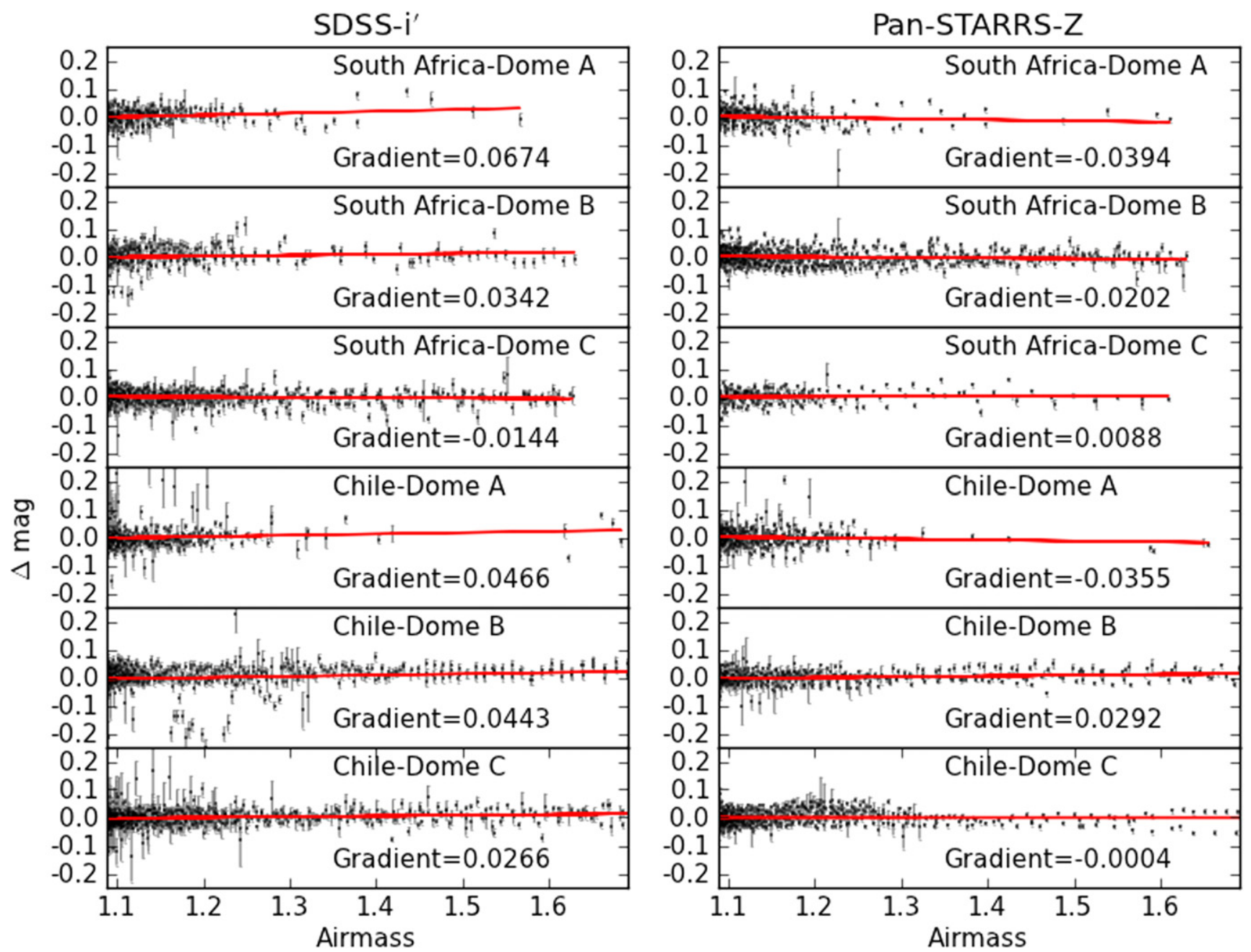

Figure 8. Differential photometry for Luhman-16 from the different telescopes, normalized with their respective means, plotted against the airmass of each measurement. The gradient of the fitted straight line is indicated for each data set.

interval. The number of data points lying in each interval is recorded.

2. $F_{j}$ was then sorted into bins according to $n_{j}$.

3. The variance of $F_{j}$ within each bin was then calculated as an estimate of the $\nu(n)$ function.

Figure 11 presents $\nu^{1 / 2}(n)$ for our reference stars and compares our data to the $n^{-1 / 2}$ relation expected for pure white noise and the function $\nu(n)=\sigma_{w}^{2} / n+\sigma_{r}^{2}$, combining red and white noise sources. We estimate the amplitude of white noise by calculating the standard deviation of the whole light curve and that of the red noise for each star by fitting the above relation to the data. $\sigma_{r}$ was found to be between 0.001 and 0.007 mag in Pan-STARRS-Z but more consistently between 0.0025 and $0.0030 \mathrm{mag}$ in SDSS-i'. We also note that $\nu(1)$ is always higher than $\sigma_{w}$. This value is computed for lone data points within duration $l$, implying that no other valid measurements were obtained around that time. This often occurs due to a local dawn at a given site or the onset of poor conditions, so we would expect higher scatter.

\section{TIME SERIES ANALYSIS}

Figures 12-14 show the data collected. The lower light curve on each plot represents the difference between the SDSS $-i^{\prime}$ and Pan-STARRS-Z light curves, computed from time-averaged bins that are 30 minutes wide, designed to make apparent any distinction in phase or amplitude of variation. It confirms that the periodic, quasi-sinusoidal variability observed by other authors occurs in our data with a similar amplitude $(\Delta m \sim$ $0.1 \mathrm{mag}$ ) in both bandpasses. In general the variability occurs in both passbands, but interestingly not always with exactly the same morphology or amplitude. It should be noted that the variability is better defined in the Pan-STARRS-Z light curve in part because the target is markedly brighter in that filter.

We initially used an implementation of the SchwarzenbergCzerny algorithm to search for periods between 0.04 and $20.6 \mathrm{~d}$ in both time series, as it is optimized for the analysis of noncontinuously sampled data sets (Schwarzenberg-Czerny 1999). The range of periods searched was set by the average sampling cadence at the short end and by half the length of the data set at the long end in order to include at least two full cycles to confirm the period. The periodograms resulting from the whole light curves are shown in Figure 15. The strongest peaks in both cases are dominated by the window function of the data sets, particularly between frequencies, $f=0-1 \mathrm{~d}^{-1}$. However, visual inspection of the light curve clearly shows periodic signals on timescales of hours, which manifest as frequency peaks around $f \sim 4.4-5.1 \mathrm{~d}^{-1}$, with aliases around $f \sim 2.297 \mathrm{~d}$ ${ }^{-1} \cong 10.45 \mathrm{hr}$. These groups of peaks with similar power indicate that there is no one single clear period occurring 

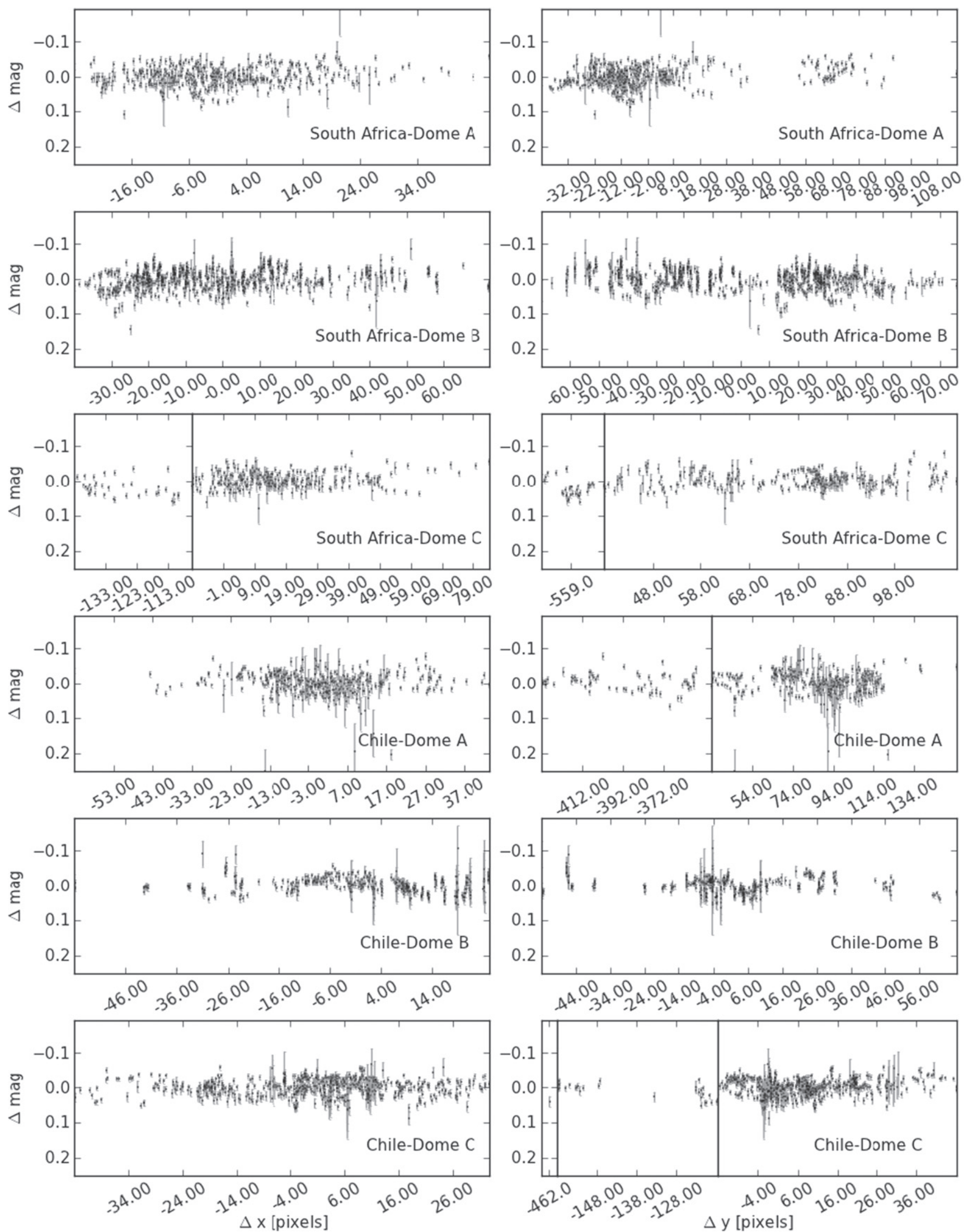

Figure 9. Magnitude residuals (Pan-STARRS-Z) for the target photometry plotted as a function of its relative CCD pixel position, separated into the different cameras. In some data sets, the pointing was substantially offset in position for some frames. These data have been included, but the white space has been eliminated for clarity. 


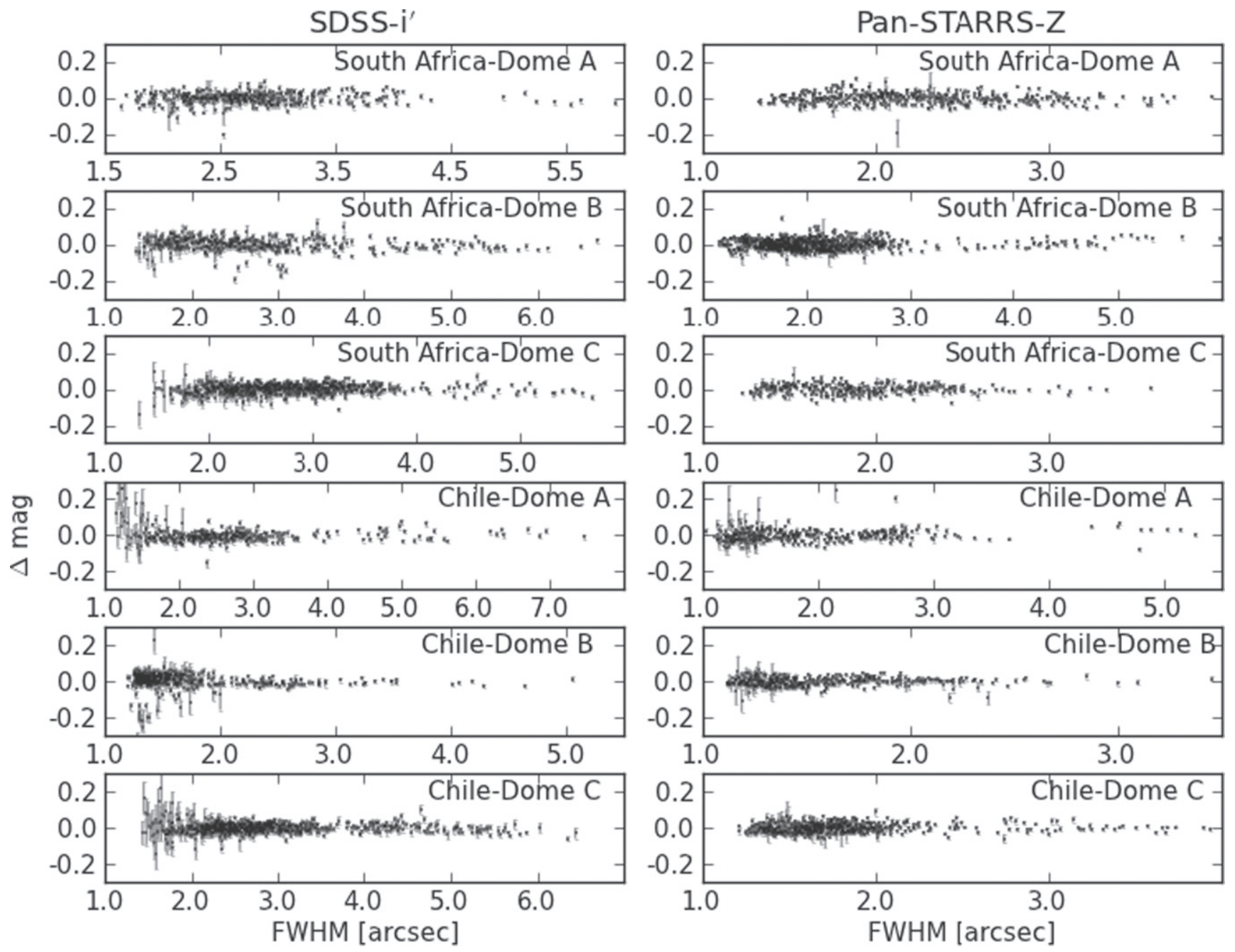

Figure 10. Differential photometry for Luhman-16 from the different telescopes, normalized with their respective means, plotted against the average FWHM of stars in the corresponding image.
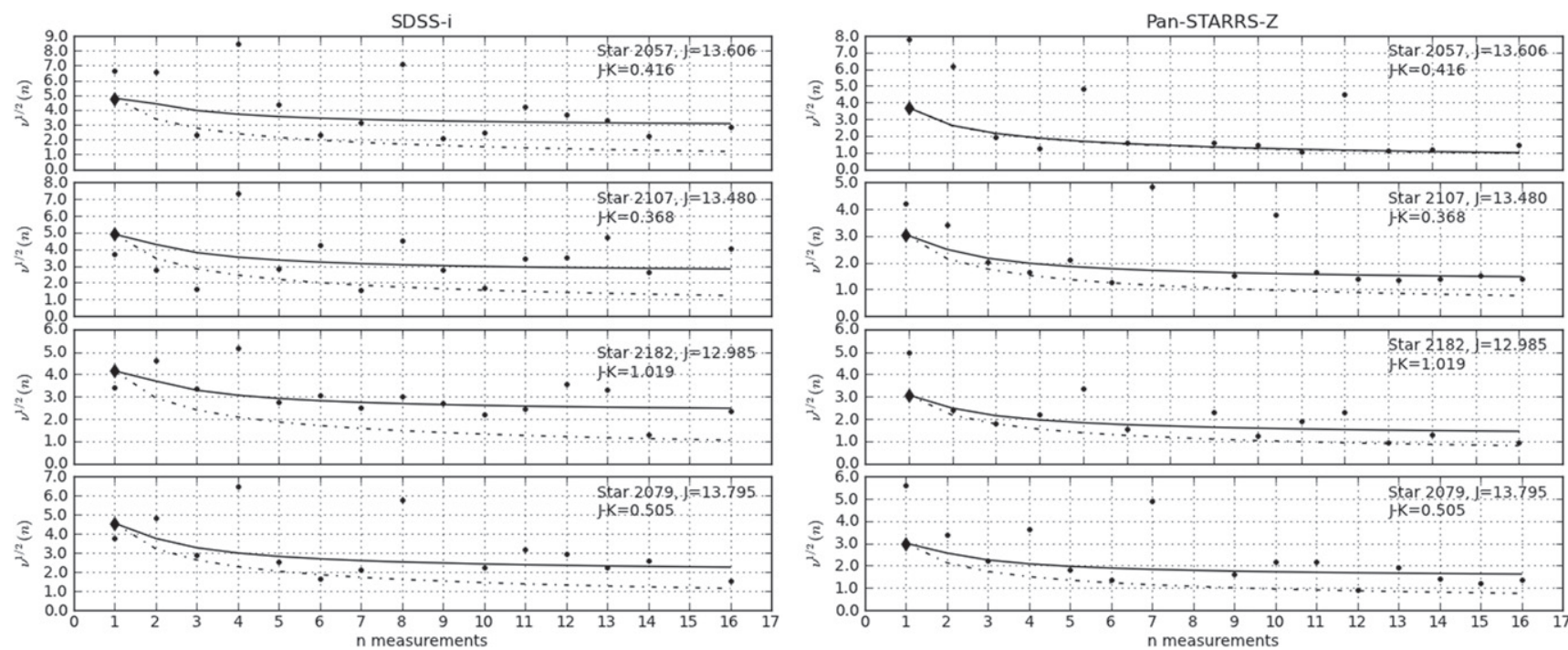

Figure 11. Plots of the $\nu(n)$ function for constant reference stars. Overplotted are functions representing pure white noise (dotted-dashed line) and a combination of red+white noise (solid line). In both cases, the white noise term is estimated from the standard deviation of the whole light curve, indicated by a diamond, while the degree of red noise was estimated by fitting this relation to the data. 

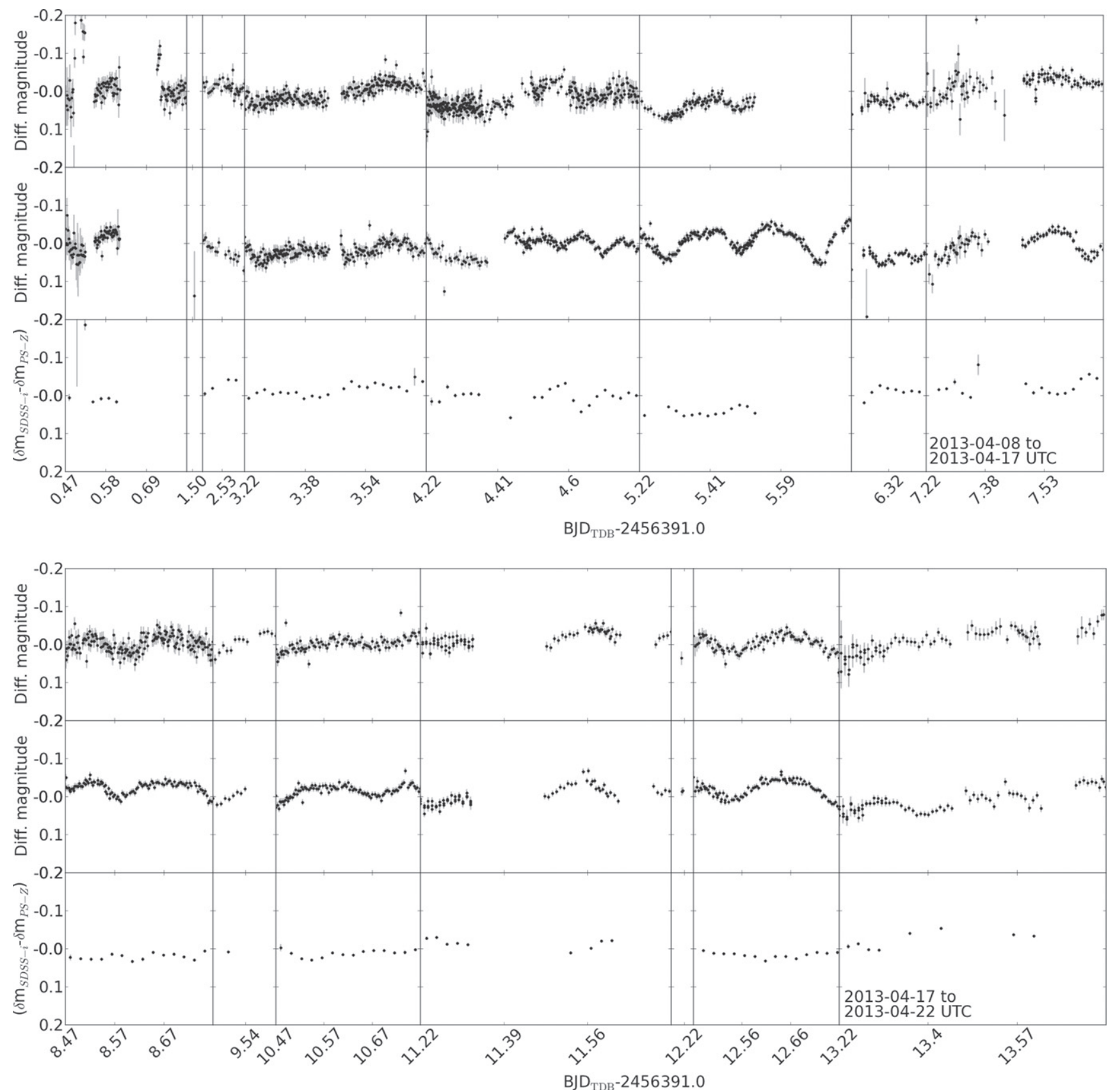

Figure 12. First two sections of the light curve of Luhman-16 in SDSS-i' (top curve) and Pan-STARRS-Z (middle). The color index ( $Z-i)$ curve is indicated in the bottom plot, binned in time with a 30-minute cadence.

consistently across the whole data set. We measured each of the peaks around these frequency bands, estimating the error on the measurements from the width of the peak, and verified each one by folding the light curve on that period and its integer multiples to identify aliases. When folded on a period of $\sim 10.45 \mathrm{hr}$, the light curves showed two minima in light, suggesting that the true period is actually half of this. We measure this period to be $5.28 \pm 0.01 \mathrm{hr}$ in both colors. However, when the whole light curves were folded on this period, there remained a fairly high degree of scatter. Some data folded cleanly on this period, but data from other nights showed quasi-sinusoidal trends offset in phase. This suggested that the ephemeris was not constant for the duration of the observations.
To investigate this, we performed a periodicity analysis of the four extended sections of the light curve that have the densest coverage spanning two sites: 2013 April 11-14, April 18-21, April 24-26, and May 10-13 UTC (see Figure 16). We measured periods independently from both the SDSS $-\mathrm{i}^{\prime}$ and Pan-STARRS-Z light curves, and the results are compared in Table 2. While most often similar, the period measured in PanSTARRS-Z is sometimes smaller.

To test the veracity of these periods, we folded the light curves on each one in turn. The most clearly defined epoch of minimum light occurred at $\mathrm{BJD}_{\mathrm{TDB}}{ }^{4}=2456403.54 \pm 0.04 \mathrm{~d}$. However, we note a high degree of asymmetry when the light

\footnotetext{
4 Barycentric Julian Date (Barycentric Dynamical Time).
} 

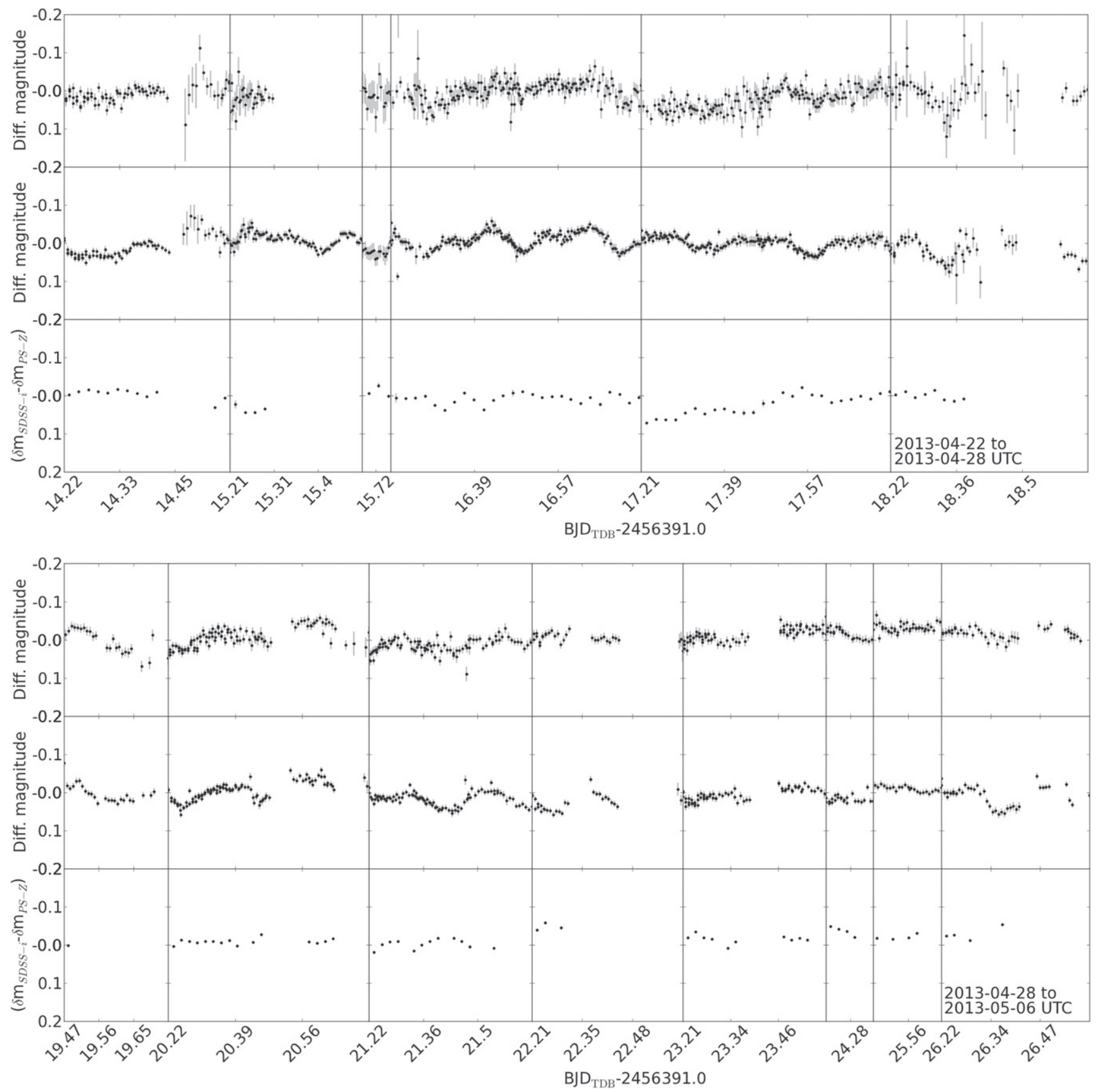

Figure 13. Middle two sections of the light curve of Luhman-16 in SDSS-i' (top curve) and Pan-STARRS-Z (middle). The color index ( $Z-i$ ) curve is indicated in the bottom plot, binned in time with a 30 -minute cadence.

curves are folded on these periods, even when data from a single night are used, so for clarity we adopted the most recent minimum-light epoch in each section during this analysis. Figures 17 and 18 show the most extended sections of the light curves in detail. Each section spans $\sim 13 \mathrm{hr}$, and $>2 \times$ the 4.87 $\pm 0.01 \mathrm{hr}$-period measured by Gillon et al. (2013), and it can be seen that the morphology of the light curves changed markedly within this timeframe.

To further test the periodicities identified, and also to explore the possibility of multiple periods, we also employed the PERIOD04 software package to analyze the Pan-STARRS-Z time series. This package, including its handling of photometric and period uncertainties, is described in Lenz \& Breger (2005). This analysis considered both the whole light curve and the sections described above, for comparison. Once the software identified each period in the data via a Fourier transform, it is removed from the time series and the residuals are searched for secondary frequencies. This procedure found evidence of multiple periods only in those light curve sections longer than 1 day (presented for comparison in Table 3). The two singlenight time series of Section 3 manifested single periods of 5.07 and $5.37 \mathrm{hr}$. We therefore interpret the apparent multiperiodicity in longer data sections to be a result of variations in the quasi-periodic photometric modulation over time rather than simultaneous frequencies. That is, the light curves consistently exhibit modulation on a period close to $5.28 \mathrm{hr}$, but which fluctuates in phase and/or cycle period.

In order to quantify the rate of change in the measured periodicities, we focused on the Pan-STARRS-Z data in light curve Section 3 (2013 April 24-26) and attempted to 

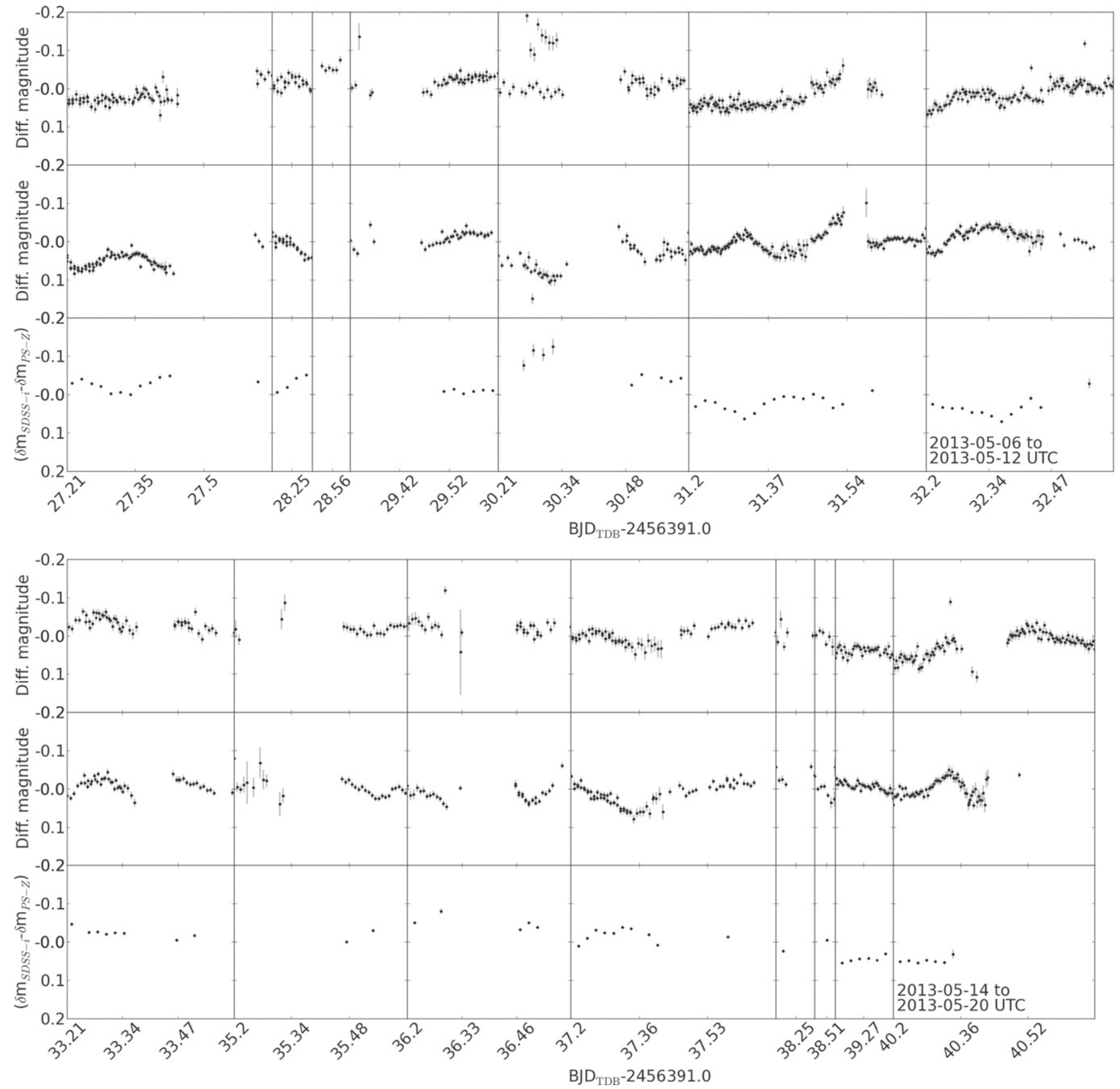

Figure 14. Final two sections of the light curve of Luhman-16 in SDSS-i' (top curve) and Pan-STARRS-Z (middle). The color index ( $Z-i$ ) curve is indicated in the bottom plot, binned in time with a 30-minute cadence.

independently measure the period from subsequent nights of data. The corresponding periodograms are compared in Figure 19 and Table 3. Data from the 24th to 25th shows a distinct rotational cycle on a frequency of $f=4.738 \mathrm{~d}^{-1}$, period $P=5.066 \mathrm{hr}$. The frequency peak is less well-defined for the data from the 25 to the 26th due to marked changes in amplitude and modulation within the same night, but is clearly offset from the earlier periodicity. The estimated frequency was $f=4.464 \mathrm{~d}^{-1}, P=5.37 \mathrm{hr}$.

We note that the presence of red noise in the photometry could in principle produce spurious periodic signatures, particularly in shorter sections of data. To test our confidence that the periods measured in the target light curve are due to intrinsic variability, we used PERIOD04 to produce periodograms for the comparison star light curves. If the periodicities detected were due to red noise, the comparison star periodograms should show a signal at the same frequency. If little or no signal is evident, we may have greater confidence that the period is intrinsic to the target. We compare the periodograms of the comparison stars with that of the target in Figure 19. By averaging together the former, we computed the amplitude of the periodogram of constant stars $\left(a_{\text {target }}(f)\right)$ at the frequencies of the periods detected in the target light curve. These are compared with the amplitude of the target's periodogram $\left(a_{\text {target }}(f)\right)$ as an amplitude ratio $=a_{\text {target }}(f) / a_{\text {target }}(f)$ in Table 3. The periodicities detected in all sections of the Luhman-16 light curve are stronger by several factors than any quasi-periodic signatures due to residual red noise. 


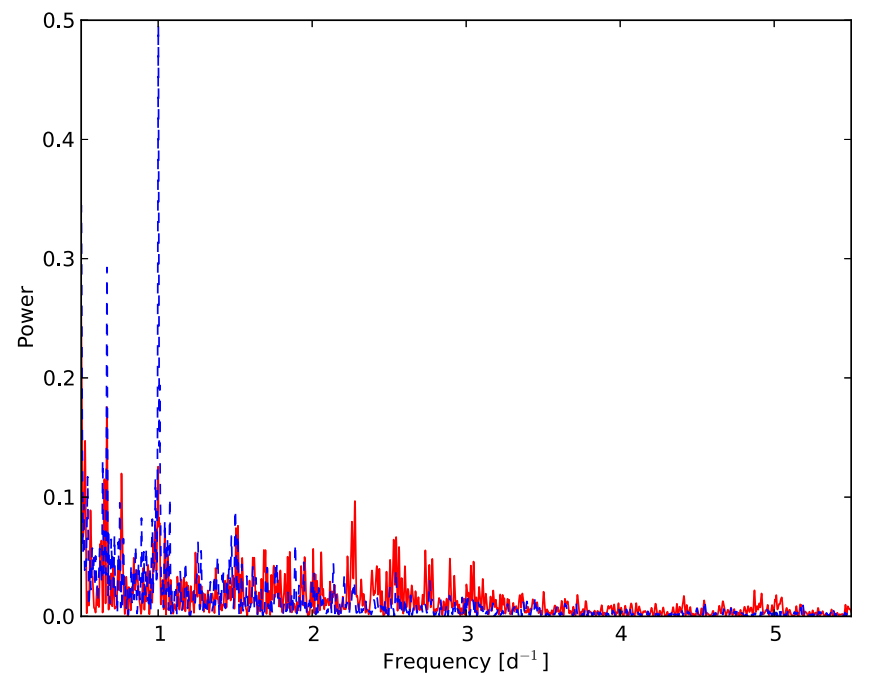

Figure 15. Periodogram analysis of the complete SDSS-i' (blue, dashed) and Pan-STARRS-Z (red, solid) light curves of Luhman-16. No significant structure is seen at $f>5.5 \mathrm{~d}^{-1}$.

Finally, we examined all sections of the light curves where simultaneous SDSS-i' and Pan-STARRS-Z data were available, looking for evidence of phase shifts between the variability in the two colors. Most commonly, the same broad trends were observed with no discernible time-lag but sometimes with a slightly lower amplitude in SDSS-i' (e.g., 2013 April 20-21, $\mathrm{BJD}_{\mathrm{TDB}}=2456403-4$, Section 2). But there were also occasions when the variability was not echoed in both light curves: 2013 May $10-11$, $\mathrm{BJD}_{\mathrm{TDB}}=2456422-3$ (Section 4) is a good example of this.

While there was some variation in the morphology of the simultaneous light curves, in general the amplitude of modulation was found to be similar in both bands: $\sim 0.05 \mathrm{mag}$ in SDSS $-\mathrm{i}^{\prime}$ and $\sim 0.06 \mathrm{mag}$ in Pan-STARRS-Z, occasionally with more dramatic features, with amplitudes up to $0.1 \mathrm{mag}$. Figures 17 and 18 highlight how quickly the amplitude can change. For example, on 2013 May 10-11, the amplitude in Pan-STARRS-Z increased from 0.043 to 0.09 mag in $4.8 \mathrm{hr}$.

\section{DISCUSSION}

From our analysis the following main characteristics of the light curve of Luhman-16 emerge.

1. The period as measured from the photometric modulation was consistently close to $5.28 \mathrm{hr}$ in both filters. However, it was observed to vary markedly, even over the course of $24 \mathrm{hr}$, and may be different in different filters. We measured apparent periods ranging between 4.464 and 5.844 $\mathrm{d}$ in Pan-STARRS-Z and 4.493-5.37 d in SDSS- $\mathrm{i}^{\prime}$ from different data sections between 1 and $3 \mathrm{~d}$ in length. We interpret this as quasi-periodic modulation rather than as multiple periodicities.

2. The amplitude in the $i^{\prime}$ - and Z-bands was generally similar, though that in the $Z$-band was sometimes slightly larger $(\sim 20 \%)$. Both are typically $\sim 0.05 \mathrm{mag}$, though modulations of up to $0.1 \mathrm{mag}$ were observed.

3. The amplitude changes rapidly, by up to a factor of $\sim 2$ over the course of one rotational cycle.
4. Generally the variability appears in both filters at the same time and we find no clear evidence for phase shifts between passbands, but note some occasions when the object is variable in Pan-STARRS-Z but not in SDSS- $\mathrm{i}^{\prime}$.

Gillon et al. (2013) were able to separate the components during a part of their monitoring campaign and show that the variations at that time are caused by the $\mathrm{T}$ component. Biller et al. (2013) confirmed that the majority of the variation is due to the T-dwarf, though they found evidence of low-amplitude variability in the L-component as well. This fits well with the other known cases of pronounced, periodic variability in the L/ $\mathrm{T}$ regime that are also found for objects with early $\mathrm{T}$ spectral types (T0.5, T1.5, T2.5, Artigau et al. 2011; Radigan et al. 2012; Girardin et al. 2013), with periods ranging between 2 and $20 \mathrm{hr}$ (Herbst et al. 2007, pp. 297-311; Reiners \& Basri 2008). It should be borne in mind that the spatially resolved monitoring has only been possible for relatively short periods of time and it is clear that the modulating surface features can change quite markedly. It remains possible that the L-component is not generally "quieter" but was simply quiescent at the time the data were obtained. However, it seems likely that the L-component undergoes lower-amplitude variations (at least in optical passbands) and therefore the following discussion assumes that our data represent variability in the cooler binary component. Significantly, Luhman-16 shows clear changes in the photometric modulation on timescales shorter than it's own average period and even more so within one (Earth) day. We observe changes in amplitude by up to a factor of two over the course of $\sim 5 \mathrm{hr}$, a behavior that is unprecedented for this type of object.

Biller et al. (2013) report an anticorrelation in variation between their (spatially resolved) $r^{\prime}, i^{\prime}$, and $z^{\prime} H$ light curves. We did not observe this phenomenon but suggest that this may be a result of the different bandpasses of " $z$ " filter used. The Pan-STARRS-Z filter used by the LCOGT $1 \mathrm{~m}$ network has a central wavelength of $869.3 \mathrm{~nm}$ and a relatively narrow 818.0-920.5 $\mathrm{nm}$ bandpass compared with the $120 \mathrm{~nm}$-wide SDSS- $z^{\prime}$ filter centered at $910 \mathrm{~nm}$. The data presented in this work sample the optical rather than NIR behavior of this object.

We next consider a number of possible causes of this variability.

\subsection{Magnetic Activity}

A large fraction of late-type $M$ dwarfs show periodic variations due to magnetically induced star spots, analogous to the solar spots (see the discussion in Scholz \& Eislöffel 2004, 2005). However, several arguments can be used to exclude this option for early T dwarfs. First, such star spots are typically stable over several weeks and many rotational cycles, over a wide range of stellar masses (Hussain et al. 2001; Scholz \& Eislöffel 2004). Second, toward late $M$ dwarfs the photometric amplitudes produced by such spots drop considerably (Scholz et al. 2009). Other indicators of magnetic activity tend to disappear in the same spectral regime (continuous X-ray- and $\mathrm{H} \alpha$ emission, Mohanty et al. 2002; Stelzer et al. 2006). For L dwarfs stable periodic variations are rare (Bailer-Jones \& Mundt 2001; Gelino et al. 2002), and apart from radio emission and transient events, signs of magnetic activity are also rarely observed (Schmidt et al. 2007; Berger et al. 2010, e.g.,), and stringent upper limits have already been placed for Luhman-16 by Osten et al. (2015). A re-appearance 

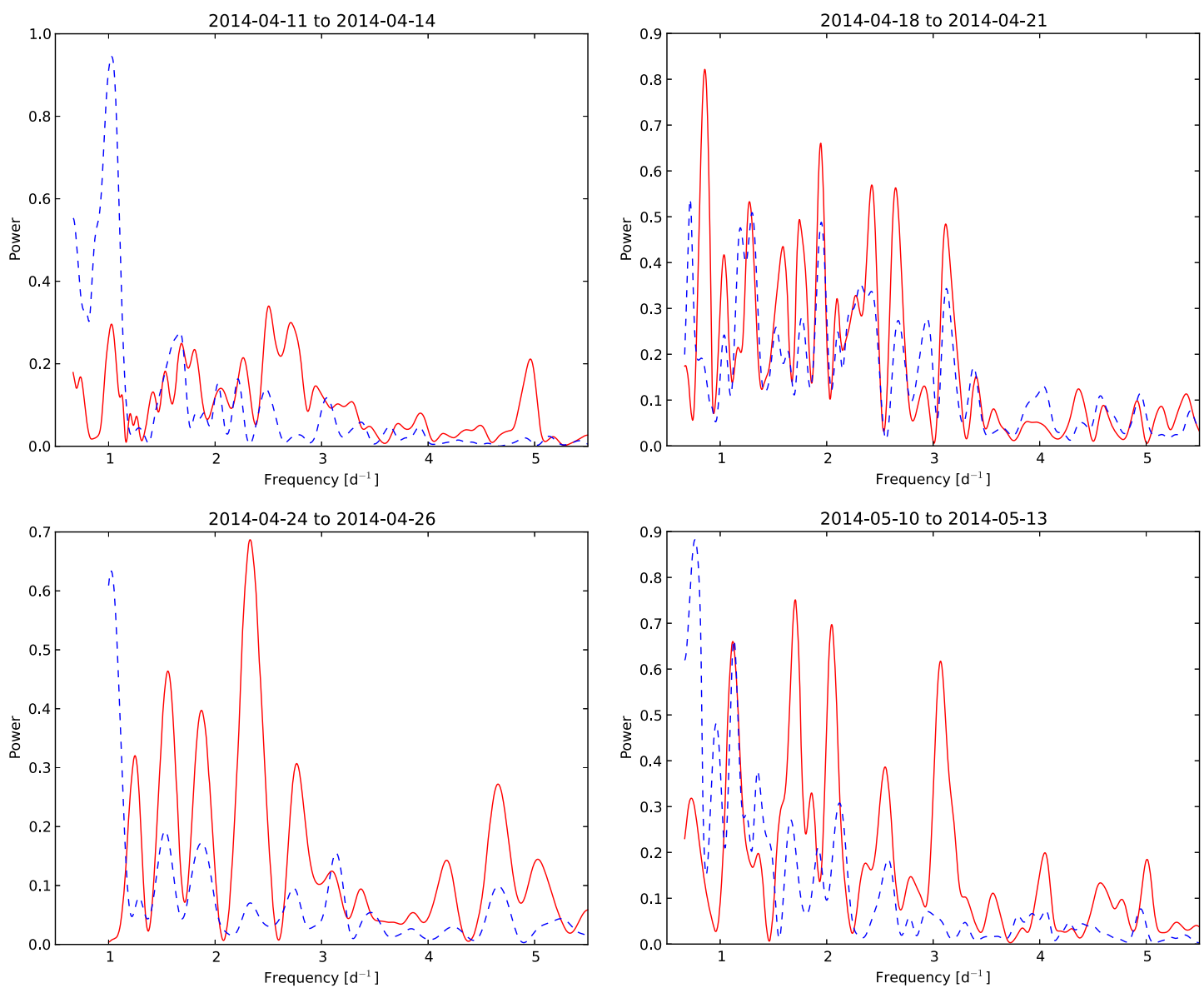

Figure 16. Periodogram analysis by the Schwarzenberg-Czerny algorithm of the four most densely sampled sections of the light curve for Luhman-16. The red solid curves are derived from the Pan-STARRS-Z light curve, while the blue dashed curves were produced from the SDSS-i' data.

Table 2

Rotational Periods Measured from Different Sections of the Light Curves Using the Schwarzenberg-Czerny Algorithm

\begin{tabular}{lccc}
\hline \hline Section & Date range (UTC) & Period (SDSS-i')(hr) & Period (PSz)(hr) \\
\hline 1 & 2013 Apr 11-14 & $4.84 \pm 0.58$ & $4.84 \pm 0.12$ \\
2 & 2013 Apr 18-21 & $4.86 \pm 0.11$ & $4.53 \pm 0.37$ \\
3 & 2013 Apr 24-26 & $5.15 \pm 0.22$ & $5.15 \pm 0.91$ \\
4 & 2013 May 10-13 & $4.85 \pm 0.09$ & $4.79 \pm 0.10$ \\
Whole & 2013 Apr 11-May 20 & $5.277 \pm 0.011$ & $5.280 \pm 0.010$ \\
\hline
\end{tabular}

of the star-like magnetically induced cool spots in the T dwarfs regime seems unlikely and is not further considered here. This does not preclude the presence of other surface features related to the interaction of the magnetic field (which still exist, as evidenced by the radio emission) and the atmospheric layers (e.g., charged clouds, see Helling et al. 2013).

\subsection{Patchy Cloud in the Atmosphere of a Rotating Brown Dwarf}

An inhomogeneous cloud level in the upper atmosphere would periodically expose deeper layers as the object rotated, causing variability in flux (Marley et al. 2010). This scenario would explain the changing amplitude as the evolution of the cloud layers over time and may also explain the apparent changes in period as clouds occurring at different latitudes. This interpretation has been supported by spectral and photometric studies of a number of early $\mathrm{T}$ dwarfs including Luhman-16 (e.g., Radigan et al. 2012; Apai et al. 2013; Buenzli et al. 2014), where models of time series spectra have ruled out the existence of cleared atmospheric "holes" in favor of regions of warmer, thinner clouds versus thicker, cooler clouds. Interesting, periodic variations have also been reported for objects of later spectral type, including the T6.5 dwarf 2MASS J22282889-4310262 (2M2228). Buenzli et al. (2012) identified significant phase shifts as a function of wavelength from NIR HST/WFC3 spectroscopy and Spitzer photometry of this object, which they interpreted as probing different pressure levels within the object's atmosphere and discuss a number of plausible models of heterogeneous cloud scenarios.

A critical question is then "what level of the atmosphere do our observations probe?" To answer this, we used a Drift- 

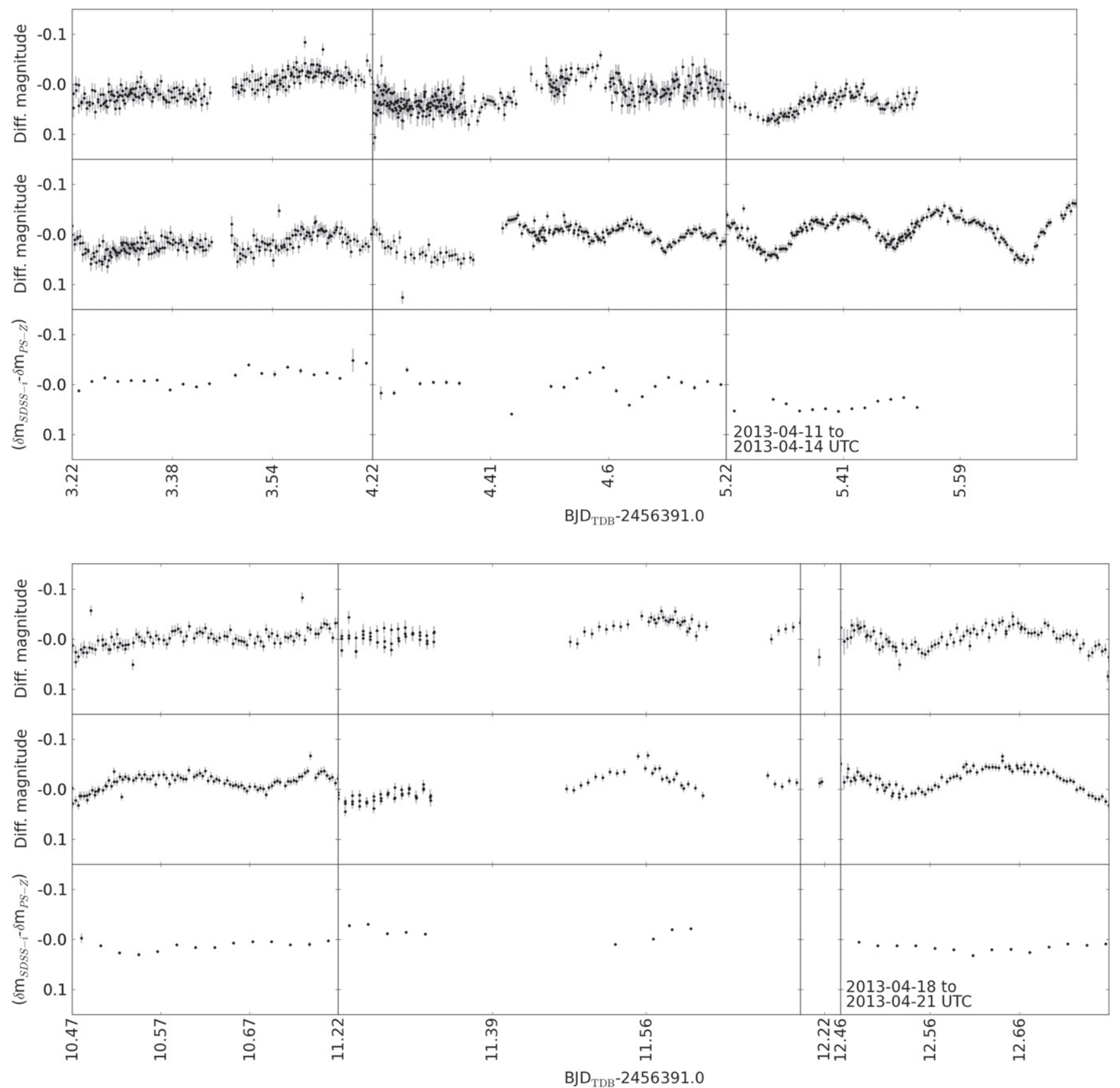

Figure 17. Close-up sections of the most densely sampled sections of the light curves, taken between 2013 April 11-14 (Section 1, top), April 18-21 (Section 2). The SDSS$\mathrm{i}^{\prime}$ data are shown in the upper curve and the Pan-STARRS-Z data are in the middle curve. The difference between the time-binned light curves is indicated in the bottom plot.

Table 3

Periodicities Measured from Different Sections of the Pan-STARRS-Z Light Curve Using PERIOD04

\begin{tabular}{|c|c|c|c|c|}
\hline Section & Date range (UTC) & Period (PSz)(hr) & Frequency $\left(\mathrm{d}^{-1}\right)$ & Amplitude Ratio \\
\hline \multirow[t]{2}{*}{$\overline{1}$} & 2013 Apr $11-14$ & $4.773 \pm 0.032$ & $5.029 \pm 0.033$ & 14.680 \\
\hline & & $4.493 \pm 0.028$ & $5.341 \pm 0.034$ & 14.985 \\
\hline \multirow[t]{2}{*}{2} & 2013 Apr $18-21$ & $4.500 \pm 0.017$ & $5.333 \pm 0.020$ & 9.621 \\
\hline & & $5.061 \pm 0.044$ & $4.742 \pm 0.041$ & 8.908 \\
\hline 3 & 2013 Apr $24-26$ & $5.112 \pm 0.016$ & $4.695 \pm 0.015$ & 14.408 \\
\hline $3 \mathrm{a}$ & 2013 Apr $24-25$ & $5.066 \pm 0.085$ & $4.738 \pm 0.079$ & 6.385 \\
\hline $3 b$ & 2013 Apr $25-26$ & $5.37 \pm 0.089$ & $4.464 \pm 0.074$ & 6.577 \\
\hline 4 & 2013 May $10-13$ & $4.716 \pm 0.011$ & $5.089 \pm 0.011$ & 22.259 \\
\hline \multirow[t]{5}{*}{ Whole $1 / \mathrm{c}$} & & $5.326 \pm 0.001$ & $4.507 \pm 0.001$ & 6.597 \\
\hline & & $5.271 \pm 0.001$ & $4.5528 \pm 0.0009$ & 12.0658 \\
\hline & & $4.744 \pm 0.0008$ & $5.0595 \pm 0.0008$ & 10.2985 \\
\hline & & $4.613 \pm 0.001$ & $5.203 \pm 0.001$ & 11.170 \\
\hline & & $4.107 \pm 0.001$ & $5.844 \pm 0.002$ & 7.206 \\
\hline
\end{tabular}

Note. The amplitude ratio compares the amplitude of the target's periodogram with that of the combined periodograms of the comparison stars at each frequency. 

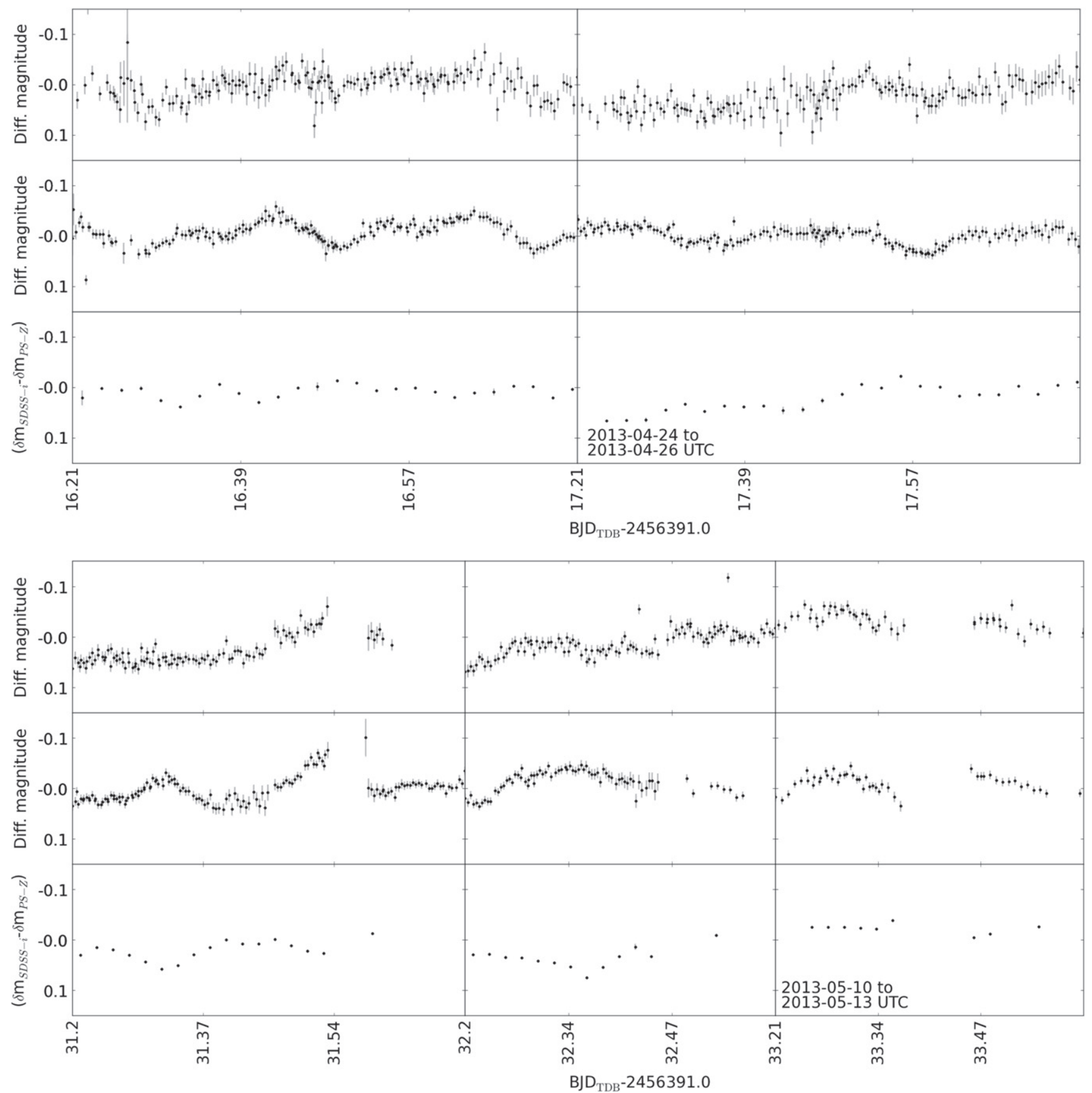

Figure 18. Close-up sections of the most densely sampled sections of the light curves, taken between April 24-26 (Section 3, top) and May 10-13 (Section 4). The SDSS-i' data are shown in the upper curve and the Pan-STARRS-Z data are in the middle curve. The difference between the time-binned light curves is indicated in the bottom plot.

Phoenix model atmosphere (Helling et al. 2008c; Witte et al. 2011) to explore the cloud opacity and the extinction as a function of wavelength for an object with $T_{\text {eff }}=1300 \mathrm{~K}$ and $\log g=5.0$. The model atmosphere included a detailed simulation of cloud formation based on a kinetic description of the microphysical processes involved (Helling \& Fomins 2013).

Figure 20 (left) demonstrates that the upper cloud levels are largely transparent for observations at the central wavelength of the filters we used. Gas opacity becomes significant at temperatures between $\sim 1200$ and $1600 \mathrm{~K}$. The cloud layers at $T_{\mathrm{gas}}=1500-1800 \mathrm{~K}$ have similar mean grain sizes but differ in composition (silicate versus iron), while for layers between
$T_{\text {gas }}=1200-1500 \mathrm{~K}$ the opposite is true (Figure 20, right). This is consistent with Buenzli et al. (2012) in that comparing different IR band (or IR with optical filter) observations would probe different levels of the atmosphere. However, we would not expect to see phase offsets between our optical light curves in this model, given the similarity of the extinction profiles in Figure 20.

We also examined the timescales needed for convective mixing, advection, and gravitational settling, applying the same model atmosphere structure as in Figure 20 (Figure 21). We found that convective mixing with overshooting is too slow $\left(\tau_{\text {mix }}>100 \mathrm{~d}\right)$ to explain our observations. The advection timescale is very small if the velocities recommended in 

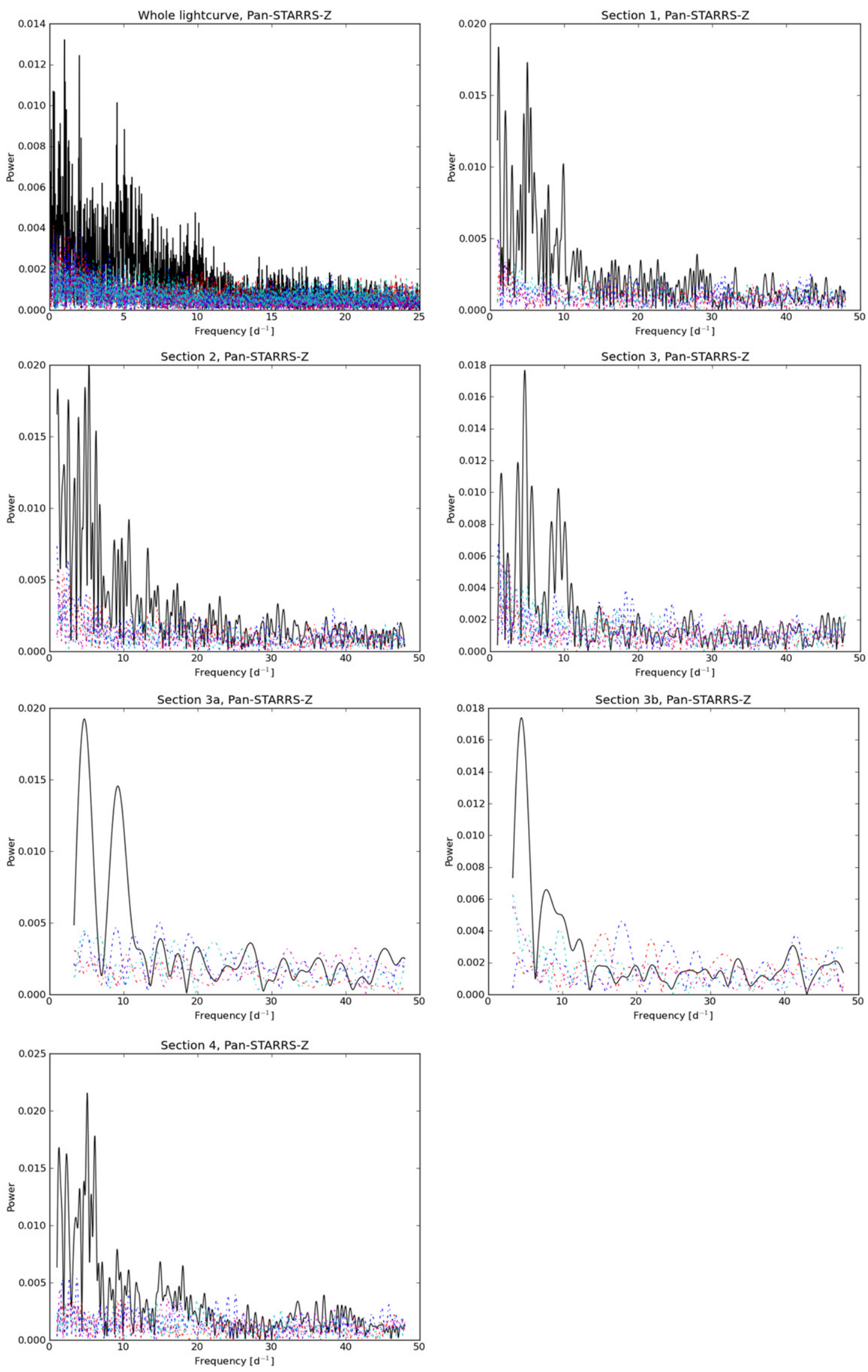

Figure 19. PERIOD04 periodogram of the Pan-STARRS-Z light curve for Luhman-16 compared with the periodograms of the comparison star light curves for the same data section: (top to bottom) whole light curve, 2013 April 11-14 (Section 1), April 18-21 (Section 2), April 24-26 (Section 3), April 24-25 (Section 3a), April 25-26 (Section 3b), and May 10-13 (Section 4). 

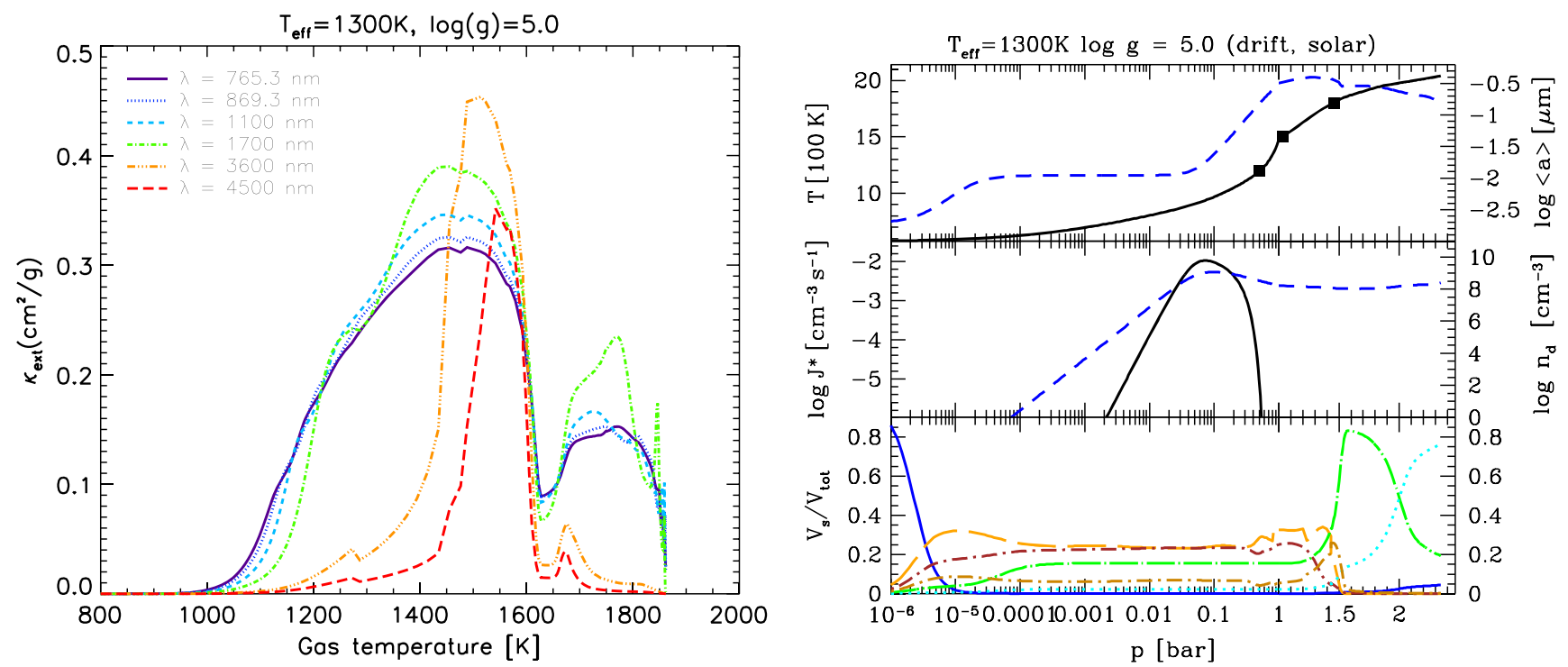

Figure 20. Drift-Phoenix model atmosphere results for $T_{\text {eff }}=1300 \mathrm{~K}$ and $\log g=5.0$. (Left): The total opacity, $\kappa_{\text {ext }}$, including absorption and scattering, due to gas in clouds as a function of pressure for observations in the optical bandpasses used in this work (SDSS-i' $\lambda_{c}=765.3 \mathrm{~nm}$, Pan-STARRS-Z $\lambda_{c}=869.3 \mathrm{~nm}$ ), with NIR filters used in other studies plotted for comparison. (Right): The cloud structure with atmospheric pressure in terms of temperature (top)/mean grain size $\langle a\rangle$, rate of formation per second and per $\mathrm{cm}^{3}$ atmospheric gas/density of dust particles (middle) and composition (bottom), where $V_{S} / V_{\text {tot }}$ indicates the amounts of the following materials as a fraction of the total: dark blue $-\mathrm{TiO}_{2}[\mathrm{~s}]$, green $-\mathrm{Fe}[\mathrm{s}]$, light blue $-\left[\mathrm{Al}_{2} \mathrm{O}_{3}\right]$, orange $-\mathrm{Mg}_{2} \mathrm{SiO}_{4}[\mathrm{~s}]$, brown $-\mathrm{SiO}$ [s], brown-orange- $\mathrm{MgO}$.

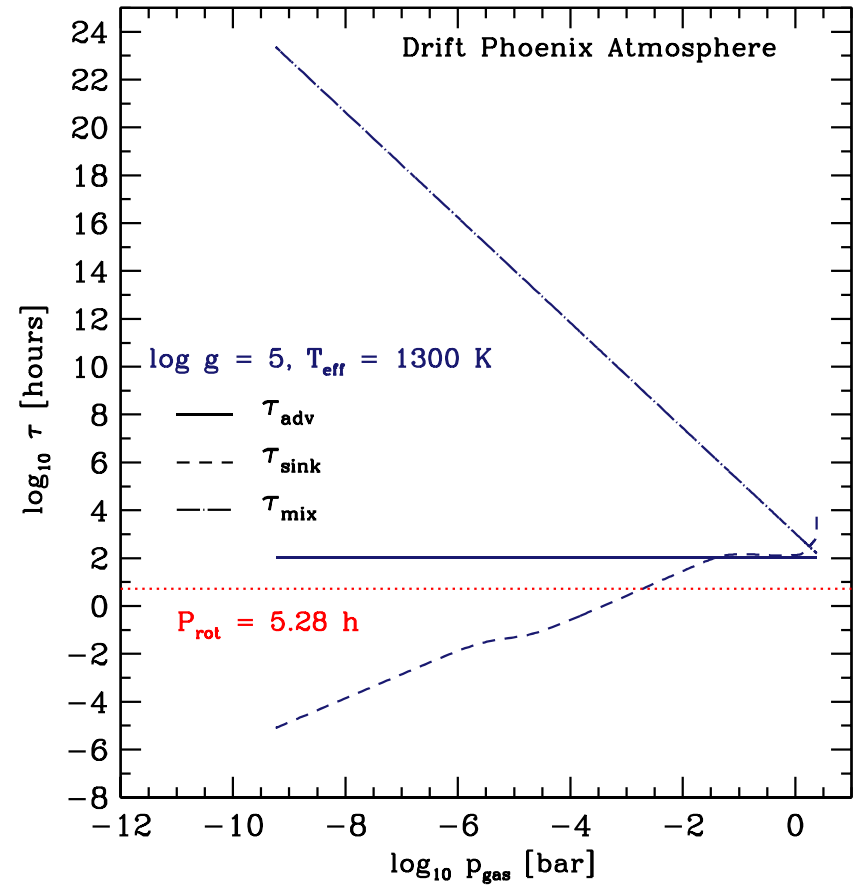

Figure 21. Advective $\left(\tau_{\text {adv }}\right)$, convective mixing $\left(\tau_{\text {mix }}\right)$, and gravitational settling $\left(\tau_{\text {sink }}\right)$ timescales evaluated for an example Drift-Phoenix model atmosphere with $T_{\text {eff }}=1300 \mathrm{~K}$ and $\log g=5.0$.

Showman \& Kaspi (2013) are adopted, although the correct length scale to use for the process is unclear. The timescale for gravitational settling depends strongly on height and grain sizes, leveling out at lower altitudes due to increasing gas density. The grain size is, however, not a parameter but the result of a kinetic dust formation model in the Drift-Phoenix model atmosphere simulations that were used here.

Our opacity calculations suggest that with some degree of mixing/rotational sheering, the lower and darker cloud layers would become visible and could be advected with the rotationally driven flow. Our simple timescale analysis further suggests that the observed variability is also connected with the cloud formation process, which is influenced by the gravitational settling. We also note that external processes can affect cloud formation: Rimmer \& Helling (2013) found that cosmic rays can impact upper cloud layers, causing a higher rate of nucleation and number of grains, and a corresponding drop in the grain size. This in turn can influence gas opacity. The cosmic ray flux will vary in space and time due to the object's magnetic field structure, as well as external conditions in the interstellar medium and the origin of the cosmic rays. Whether this is a factor in the atmosphere of Luhman-16 remains an open question; it would be very interesting to measure a magnetic field on this object. Further work is necessary to incorporate cosmic ray processes into present cloud models.

This research has made use of the LCOGT Telescope Network, and the LCOGT Archive, which is operated by the California Institute of Technology, under contract with the Las Cumbres Observatory. K.H. is supported by a Royal Leverhulme Trust Senior Research Fellowship and by the UK Science and Technology Facilities Council (STFC) grant ST/J001651/1. C.H. and G.L. highlight the financial support of the European Community under the FP7 by the ERC starting grant 257431. This research has made use of NASA's Astrophysics Data System Bibliographic Services.

Facility: LCOGT.

\section{REFERENCES}

Ackerman, A. S., \& Marley, M. S. 2001, ApJ, 556, 872

Allard, N. F., Allard, F., Hauschildt, P. H., Kielkopf, J. F., \& Machin, L. 2003, A\&A, 411, 473

Apai, D., Radigan, J., Buenzli, E., et al. 2013, ApJ, 768, id.121

Arellano Ferro, A., Bramich, D. M., Figuera Jaimes, R., Giridhar, S., \& Kuppuswamy, K. 2012, MNRAS, 420, 1333

Artigau, É., Doyon, R., Lafreniére, D., et al. 2006, ApJL, 651, L57

Artigau, É., Bouchard, S., Doyon, R., \& Lafrenié, D 2009, ApJ, 701, 1534 
Artigau, É, Lafreniére, D., Doyon, R., et al. 2011, ApJ, 739, id.48 Bailer-Jones, C. A. L., \& Mundt, R. 2001, A\&A, 367, 218

Barman, T. S., Macintosh, B., Konopacky, Q. M., \& Marois, C. 2011a, ApJ, 735, id.L39

Barman, T. S., Macintosh, B., Konopacky, Q. M., Marois, C., et al. 2011b, ApJ, 733, id.65

Bartlett, M. S. 1948, Natur, 161, 686

Berger, E., Basri, G., Fleming, T. A., et al. 2010, ApJ, 709, 332

Biller, B. A., Crossfield, I. J. M., Mancini, L., et al. 2013, ApJ, 778, 10

Bramich, D. 2008, MNRAS, 386, L77

Brown, T., Baliber, N., Bianco, F. B., et al. 2013, PASP, 125, 1031

Buenzli, E., Apai, D., Morley, C. V., et al. 2012, ApJL, 760, L31

Buenzli, E., Apai, D., Radigan, J., Reid, I. N., \& Flateau, D. 2014, ApJ, 782, 77

Burgasser, A., Marley, M. S., Ackerman, A. S., et al. 2002, ApJL, 571, L151

Burgasser, A. J., Sheppard, S. S., \& Luhman, K. L. 2013, arXiv:1303.7283

Burgasser, A. J., Gillon, M., Faherty, J. K., et al. 2014, ApJ, 785, 48

Burrows, A., Sudarsky, D., \& Hubeny, I. 2006, ApJ, 640, 1063

Cavanagh, B., Jenness, T., Economou, F., \& Currie, M. J. 2008, AN, 329, 295

Crossfield, I. J. M., Biller, B., Schlieder, J. E., et al. 2014, Natur, 505, 654

Epchtein, N., Deul, E., Derriere, S., et al. 1999, A\&A, 349, 236

Faherty, Jk., Tinney, C. G., Skemer, A., et al. 2014, ApJ, 790, 90

Gelino, C. R., Marley, M. S., Holtzman, J. A., Ackerman, A. S., \& Lodders, K. 2002, ApJ, 577, 433

Gillon, M., Triaud, A. H. M. J., Jehin, E., et al. 2013, A\&A, 555, 5

Girardin, F., Artigau, É, \& Doyon, R. 2013, ApJ, 767, id.61

Heinze, A., Metchev, S., Apai, D., et al. 2013, ApJ, 767, 173

Heinze, A., Metchev, S., Kellogg, K., et al. 2015, ApJ, 801, 104

Helling, C., \& Fomins, A. 2013, RSPTA, 371, 20110581

Helling, Ch., Ackerman, A., Allard, F., et al. 2008a, MNRAS, 391, 1854

Helling, Ch., Woitke, P., Thi, W.-F., et al. 2008b, A\&A, 485, 547

Helling, Ch., Dehn, M., Woitke, P., \& Hauschildt, P. H. 2008c, ApJL, 675, L105

Helling, Ch., Jardine, M., Stark, C., \& Diver, D. 2013, ApJ, 767, 136

Herbst, W., Eislöffel, J., Mundt, R., \& Scholz, A. 2007, in Protostars and

Planets V, Vol. 951, ed. B. Reipurth, D. Jewitt, \& K. Keil (Tucson, AZ:

Univ. Arizona Press), 297

Hussain, G. A. J., Jardine, M., \& Collier Cameron, A. 2001, MNRAS, 322, 681

Ishihara, D., Onaka, T., Kataza, H., et al. 2010, A\&A, 514, A1

Kniazev, A. Y., Vaisanen, P., Muzić, K., et al. 2013, ApJ, 770, id.124

Koen, C. 2013, MNRAS, 429, 652

Kovács, G., Bakos, G., \& Noyes, R. W. 2005, MNRAS, 356, 557
Lenz, P., \& Breger, M. 2005, CoAst, 146, 53

Lucas, P. W., Tinney, C. G., Burningham, B., et al. 2010, MNRAS, 408, L56

Luhman, K. L. 2013, arXiv:1303.2401

Mamajek, E. E. 2013, arXiv:1303.5345

Marley, M. S., Saumon, D., \& Goldblatt, C. 2010, ApJL, 723, L117

McCaughrean, M. J., Close, L. M., Scholz, R.-D., et al. 2004, A\&A, 413, 1029

Mohanty, S., Basri, G., Shu, F., Allard, F., \& Chabrier, G. 2002, ApJ, 571, 469

Oppenheimer, B. R., Baranec, C., Beichman, C., et al. 2013, ApJ, 768, 24

Osten, R. A., Melis, C., Stelzer, B., et al. 2015, ApJ, 805, 3

Pont, F., Zucker, S., \& Queloz, D. 2006, MNRAS, 373, 231

Radigan, J., Jayawardhana, R., Lafreniére, D., et al. 2012, ApJ, 750, 105

Radigan, J., Jayawardhana, R., Lafreniére, D., et al. 2013, ApJ, 778, 36

Reiners, A., \& Basri, G. 2008, ApJ, 684, 1390

Rhines, P. 1970, GApFD, 1, 273

Rimmer, P. B., \& Helling, C. 2013, ApJ, 774, 108

Schmidt, S. J., Cruz, K. L., Bongiorno, B. J., Liebert, J., \& Reid, I. N. 2007, AJ, 133, 2258

Scholz, A., \& Eislöffel, J. 2004, A\&A, 419, 249

Scholz, A., \& Eislöffel, J. 2005, A\&A, 429, 1007

Scholz, A., Eislöffel, J., \& Mundt, R. 2009, MNRAS, 400, 1548

Scholz, A., et al. 2011, in Proc. of Stellar Clusters \& Associations: A RIA

Workshop on Gaia, ed. E. J. Alfaro Navarro, A. T. Gallego Calvente \& M. R. Zapatero Osorio (Granada: IAA-CSIC), 250

Schwarzenberg-Czerny, A. 1999, ApJ, 516, 315

Showman, A. P., \& Kaspi, Y. 2013, ApJ, 776, 85

Skemer, A. J., Close, L. M., Szücs, L., et al. 2011, ApJ, 732, 107

Skrutskie, M., Cutri, R. M., Stiening, R., et al. 2006, AJ, 131, 1163

Smith, J. C., Stumpe, M. C., Van Cleve, J. E., et al. 2012, PASP, 124, 1000

Stelzer, B., Schmitt, J. H. M. M., Micela, G., \& Liefke, C. 2006, A\&A, 460, L35

Stetson, P. B. 1987, PASP, 99, 191

Tamuz, O., Mazeh, T., \& Zucker, S. 2005, MNRAS, 356, 1466

Tinney, C. G., Burgasser, A. J., \& Kirkpatrick, J. D. 2003, AJ, 126, 975

Tsuji, T., Ohnaka, K., Aoki, W., \& Nakajima, T. 1996, A\&A, 308, 29

Vaughan, S. 2012, RSPTA, 371, 20110549

Witte, S., Helling, C., \& Hauschildt, P. H. 2009, A\&A, 506, 1367

Witte, S., Helling, Ch., Barman, T., Heidrich, N., \& Hauschildt, P. H. 2011, A\&A, 529, A44

Woitke, P., \& Helling, C. 2003, A\&A, 339, 297

Wright, E. L., Eisenhardt, P. R. M., Mainzer, A. K., et al. 2010, AJ, 140, 1868 